Article

\title{
Experimental Investigation of Flow-Induced Motion and Energy Conversion of a T-Section Prism
}

\author{
Nan Shao, Jijian Lian, Guobin Xu, Fang Liu (D), Heng Deng, Quanchao Ren and Xiang Yan *(1) \\ State Key Laboratory of Hydraulic Engineering Simulation and Safety, Tianjin University, No. 92, \\ Wei Jin Road, Nan Kai District, Tianjin 300072, China; shaonan@tju.edu.cn (N.S.); tju_luntan@126.com (J.L.); \\ xuguob@sina.com (G.X.); fangliu@tju.edu.cn (F.L.); d_heng@yeah.net (H.D.); 2014205258@tju.edu.cn (Q.R.) \\ * Correspondence: xiangyan@tju.edu.cn; Tel.: +86-22-2740-1127
}

Received: 25 June 2018; Accepted: 2 August 2018; Published: 6 August 2018

\begin{abstract}
Flow-induced motion (FIM) performs well in energy conversion but has been barely investigated, particularly for prisms with sharp sections. Previous studies have proven that T-section prisms that undergo galloping branches with high amplitude are beneficial to energy conversions. The FIM experimental setup designed by Tianjin University (TJU) was improved to conduct a series of FIM responses and energy conversion tests on a T-section prism. Experimental results are presented and discussed, to reveal the complete FIM responses and power generation characteristics of the $\mathrm{T}$-section prism under different load resistances and section aspect ratios. The main findings are summarized as follows. (1) Hard galloping (HG), soft galloping (SG), and critical galloping (CG) can be observed by varying load resistances. When the load resistances are low, HG occurs; otherwise, SG occurs. (2) In the galloping branch, the highest amplitude and the most stable oscillation cause high-quality electrical energy production by the generator. Therefore, the galloping branch is the best branch for harvesting energy. (3) In the galloping branch, as the load resistances decrease, the active power continually increases until the prism is suppressed from galloping to a vortex-induced vibration (VIV) lower branch with a maximum active power $P_{\text {harn }}$ of $21.23 \mathrm{~W}$ and a maximum $\eta_{\text {out }}$ of $20.2 \%$. (4) Different section aspect ratios $(\alpha)$ can significantly influence the FIM responses and energy conversions of the T-section prism. For small aspect ratios, galloping is hardly observed in the complete responses, but the power generation efficiency $\left(\eta_{\text {out }, 0.8}=27.44 \%\right)$ becomes larger in the galloping branch.
\end{abstract}

Keywords: flow-induced motion; sharp sections; T-section prism; load resistances; section aspect ratios; energy conversion

\section{Introduction}

The flow-induced motion (FIM) phenomenon [1] widely exists in the civil engineering field, and it can lead to the failure of oscillating structures such as solar receiver tubes [2-4], long-span bridges [5], parallel twin bridges [6], offshore risers [7], and aircraft [8]. Many early studies have devoted great efforts to the suppression [9,10] of FIM. With the study of FIM increasing significantly [11-14], researchers [15-17] are gradually paying more attention to the potential of FIM energy. Many creative structures [18-20] have been proposed for exploiting this energy, especially for the vortex-induced vibration (VIV) [21] and galloping [22,23] responses of FIM.

VIV occurs due to the alternating shedding of vortices from either side of the bluff cylinder [1]. Galloping occurs due to the forces acting on a prism as it is subjected to periodic variations in the angle of attack of the flow [24]. An isolated smooth circular cylinder can only undergo VIV, while galloping is rarely observed [25]. On the contrary, galloping is easily observed for non-circular-section prisms such as rectangular prisms, triangular prisms, and passive turbulence control (PTC) circular cylinders, etc. 
There are some studies on rectangular prisms [26-29] to measure the aerodynamic forces during unsteady galloping oscillations, and some studies report that the incident angle has a significant influence on the performance of prisms. Research on triangular prisms has concluded that the stability of galloping strongly depends on the incident flow orientation and on the aspect ratio, as well as on the mass and damping [30-33]. In addition, two different types of galloping, soft galloping (SG) and hard galloping (HG), were first discovered during trials on PTC circular cylinders by Park [21]. SG is self-excited by VIV, and HG cannot be self-excited by VIV, but HG can be excited by external excitation at a high-flow velocity. Furthermore, Zhang, J. et al. [34,35] also discovered the phenomenon of SG and HG in a study on triangular prisms, and pointed out two HG types: I-type HG and II-type HG. Subsequently, Lian, J. et al. [36] found that the VIV and galloping of the triangular prism in complete FIM responses depended on the damping, stiffness, and mass of the system, as well as the section of the prism.

Research on VIV and galloping with energy conversion targets has developed rapidly in recent years. FIM power generation equipment such as VIVACE (Vortex-Induced Vibration for Aquatic Clean Energy) was successively proposed and gradually optimized. By inventing the virtual damper spring system $[37,38]\left(\mathrm{V}_{\mathrm{CK}}\right)$ and the PTC [39], Bernitsas et al., in the University of Michigan, carried out many experiments to further understand FIM responses and power generation for circular cylinders at high damping [37], a high Reynolds number [21,39,40], variable stiffness [41,42], and variable mass [41], etc. It was found that a high Reynolds number, high damping, and low mass helped to improve power generation capacity and oscillation strength. In addition, non-cylindrical oscillators such as PTC cylinders performed with outstanding advantages in power generation due to the high amplitude of galloping.

At present, most researchers pay more attention to oscillators with regular cross-sections, such as cylinders, triangular prisms, square prisms, and rectangular prisms. However, less attention is paid to oscillators with irregular cross-sections, such as the T-section. Recently, FIM tests on T-section prisms were conducted in Tianjin University [43]; the published study reported that T-section prisms also presented galloping responses. Actually, the T-section prism can be regarded as a simplified triangular prism with axisymmetric structures. Until now, previous studies on the FIM of T-section prisms have not been systematic, and have only focused on oscillation responses. The complete FIM responses and the power generation characteristics of T-section prisms have not been investigated systematically. In order to better understand the complete FIM responses and their power generation characteristics, a series of tests were conducted, specifically including the following three aspects:

(1) Experimental research on FIM responses to external forces were conducted to determine the complete FIM responses of T-section prisms;

(2) FIM power generation tests with different load resistances were carried out to investigate the power generation of the T-section prism in complete FIM responses;

(3) In order to guide the optimization design of the prism, FIM responses and power generation tests at different section aspect ratios were conducted.

\section{Experiment Setup}

\subsection{Water Channel and Calibration of Flow Velocity}

All experiments were conducted in a recirculating water channel at the State Key Laboratory of Hydraulic Engineering Simulation and Safety (SKL-HESS) of Tianjin University (shown in Figure 1). The recirculating water channel consisted of a water tank, a variable frequency power pump, a 2-m wide flow channel, a bend flow channel, a contraction section, and a 1-m wide flow channel. The whole length was about $50 \mathrm{~m}$. The water tank dimensions were $5 \mathrm{~m}$ (length) $\times 5 \mathrm{~m}$ (width) $\times 2 \mathrm{~m}$ (height) The channel was made out of transparent tempered glass and it was powered by a $90-\mathrm{kW}$ variable frequency power pump with a maximum speed of $490 \mathrm{r} / \mathrm{min}$. It could recirculate 200 cubic meters of fresh water at flow rates up to $2600 \mathrm{~L} / \mathrm{s}$. The frequency range of water pump was $0.0-50.0 \mathrm{~Hz}$, 
controlled by a frequency conversion controller (FCC). In the 1-m wide flow channel, the velocity variation range was $0.0-1.8 \mathrm{~m} / \mathrm{s}$. The water depth was $1.34 \mathrm{~m}$.

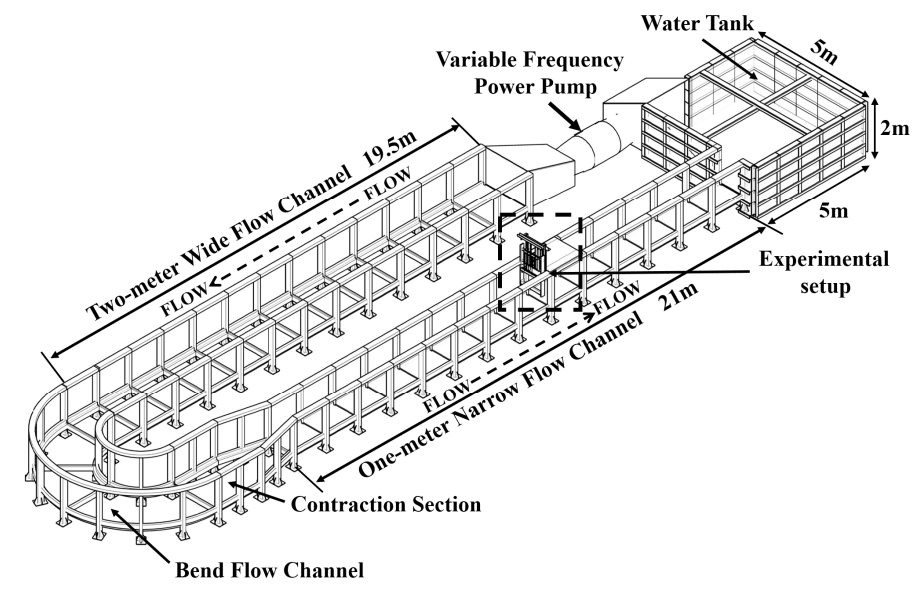

Figure 1. Recirculating water channel system.

In order to guarantee the authenticity of the results, two test instruments, a Pitot tube with a differential pressure transmitter and a propeller current meter [36], were employed to measure the flow velocity. The accuracy of the differential pressure transmitter was within $\pm 0.1 \%$ of $6 \mathrm{KPa}$, which was the linear available range, and the resolution was within $0.01 \%$ FS (Full Scale). All of the data were recorded over a time interval of $60 \mathrm{~s}$ at a $40 \mathrm{~Hz}$ sampling rate. The probes of the Pitot tube and the propeller were placed $1 \mathrm{~m}$ in front of the T-section prism.

All experiments were conducted using the TrSL3 $(20,000<\operatorname{Re}<300,000)$ flow regime [13]. This study covered a range of Reynolds numbers of $45,133 \leq R e \leq 116,396(0.516 \mathrm{~m} / \mathrm{s} \leq \mathrm{U} \leq$ $1.332 \mathrm{~m} / \mathrm{s}$ ). In order to describe the incoming flow, the flow velocity and the turbulence were both analyzed. We conducted three tests with different flow velocities. The velocity profile and the turbulence profile of the recirculating water channel were taken at a 15-100-cm deep area of the test section, as shown in Figure 2. In Figure 2a, the flow velocity near the bottom was smaller, due to viscous action. The average flow velocities in the vertical direction of three tests were $0.62 \mathrm{~m} / \mathrm{s}$, $0.84 \mathrm{~m} / \mathrm{s}$, and $1.22 \mathrm{~m} / \mathrm{s}$, respectively. The difference between the flow velocities in the oscillation range $(40 \mathrm{~cm}-80 \mathrm{~cm})$ was small. In Figure $2 \mathrm{~b}$, the turbulence grew as the flow velocity decreased and the depth increased. The average turbulences in the vertical direction of the three tests were $8 \%, 14 \%$, and $24 \%$, respectively. The difference in the turbulence in the oscillation range $(40 \mathrm{~cm}-80 \mathrm{~cm})$ was small as well. In a word, it could be ensured that the incoming flow in the oscillation range of the prism was a uniform flow.

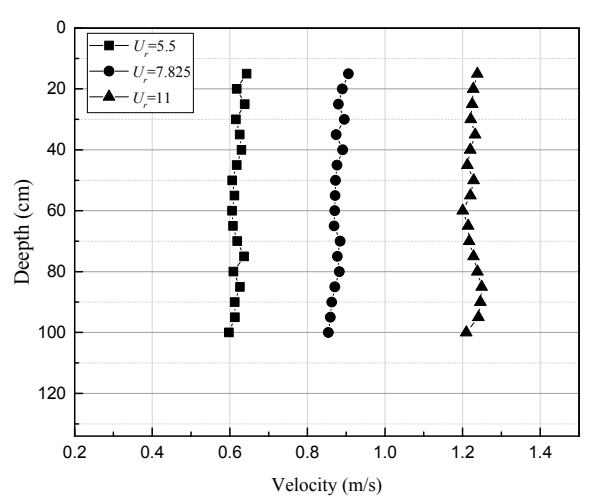

(a)

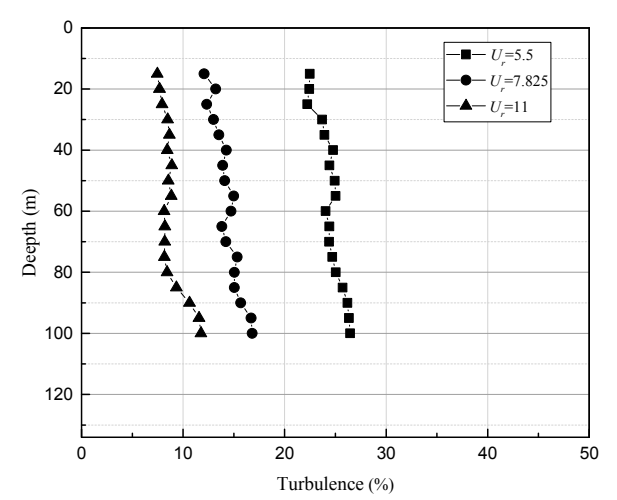

(b)

Figure 2. Incoming flow characteristics. (a) Velocity profile; (b) turbulence profile. 


\subsection{Test Apparatus and Energy Conversion System}

\subsubsection{Test Apparatus}

The test apparatus consisted of two parts: the oscillation system and the transmission system. The oscillation system included the frame, linear guide ways, side struts, spring carrier structure, and springs (shown in Figure 3). The frame was made of steel and was fixed in a moving car at the top of the 1-m wide flow channel. The linear guideways were attached onto the steel frame, parallel to the side struts and perpendicular to the flow velocity direction.

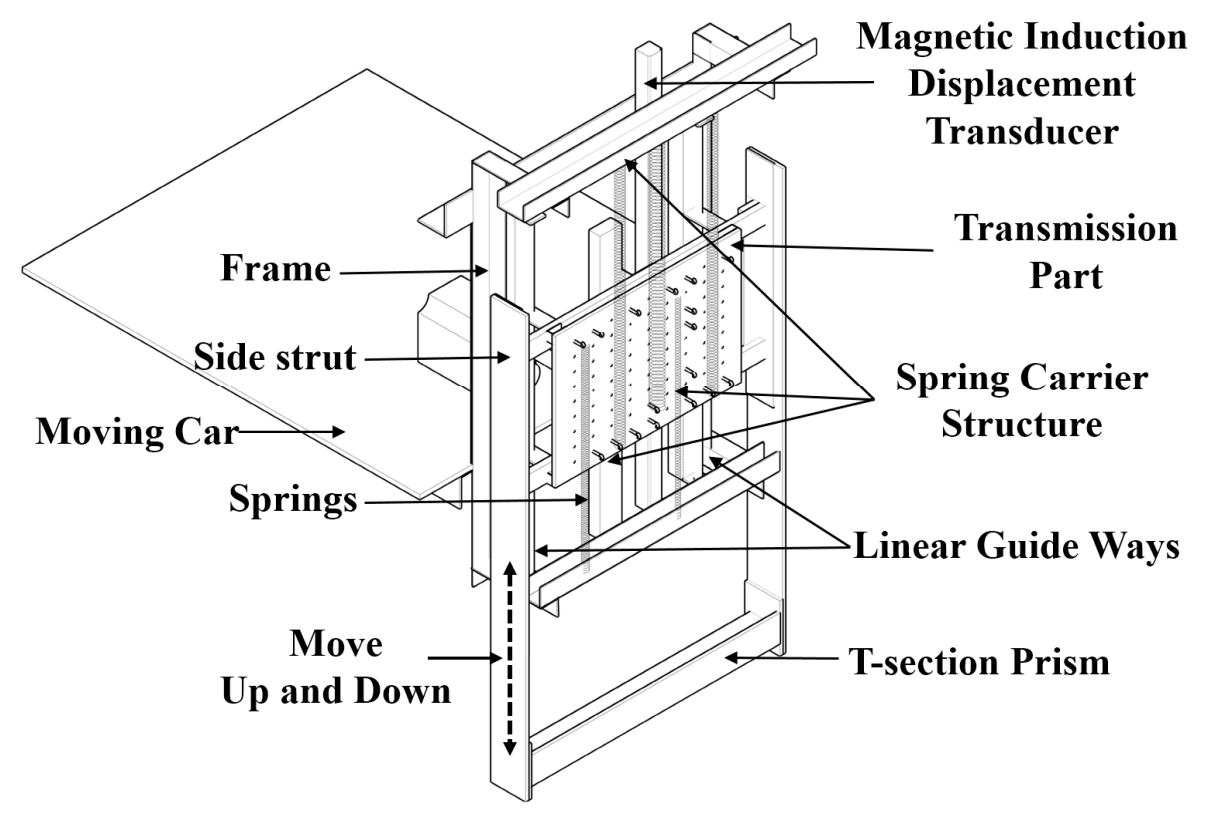

Figure 3. Test apparatus.

The transmission system included two side struts and a connective structure. The side struts, which were made of duralumin plate, were rigidly connected with the prism, which was immersed in the water. The connective structure was joined with the side struts and springs, and it was constrained to move on the linear guideways in a vertical direction by four linear bearings. Each side of the upper and the lower extensional springs was suspended vertically on the frame and the connection structure by the spring carrier structure.

\subsubsection{Energy Conversion System}

The rack was fixed into the transmission part that was connected to the rotor of the generator by the gears (shown in Figure 4). The linear motion of the prism was transferred to the rotational motion of the rotor. The generator was connected to the load resistances by the output wire, creating an electrical circuit. In this system, the mechanical energy of the prism was partly transformed to the electric energy of the generator and was dissipated by the load resistances. 


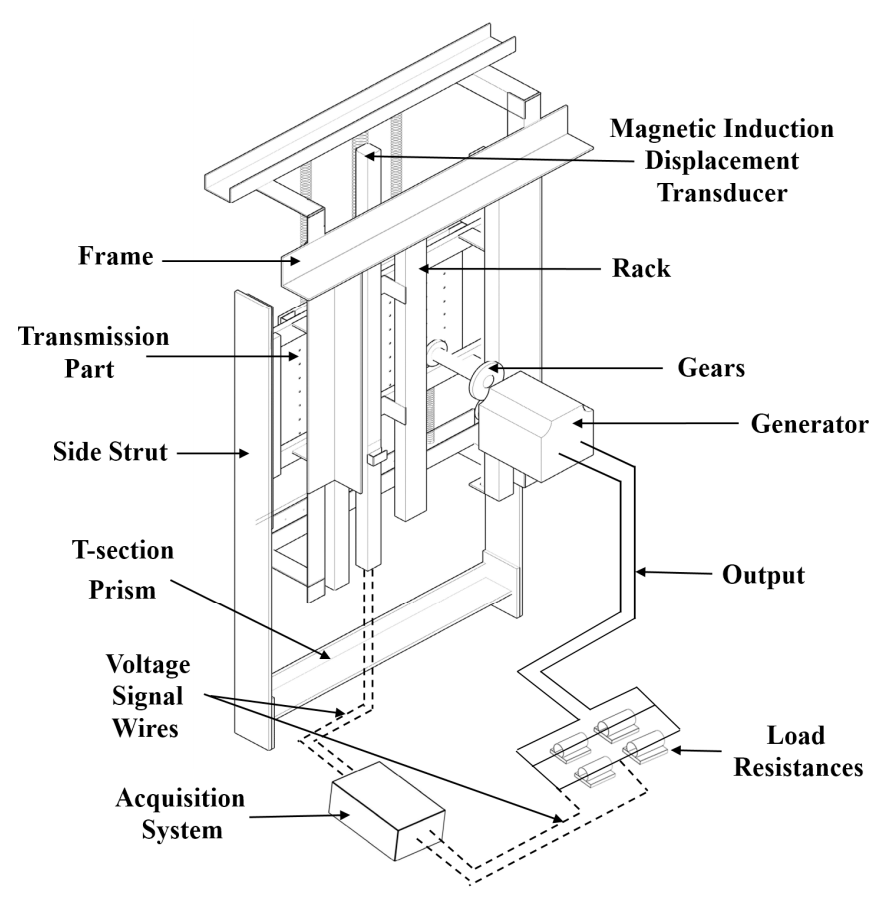

Figure 4. Energy conversion system.

\subsubsection{T-Section Prisms}

In the tests, the projection width of the T-section prism in the direction of the incoming flow $(D)$ was $0.1 \mathrm{~m}$, the prism length $(L)$ was $0.9 \mathrm{~m}$, the height of the prism cross-section $(H)$ was $0.1 \mathrm{~m}$, and the thickness $(d)$ was $0.01 \mathrm{~m}$. The prism was made of polymethyl methacrylate. A rectangular endplate was installed at both ends of the prism to reduce the effect of the boundary [30]. The thickness of the endplate was $0.01 \mathrm{~m}$ (shown in Figure 5).

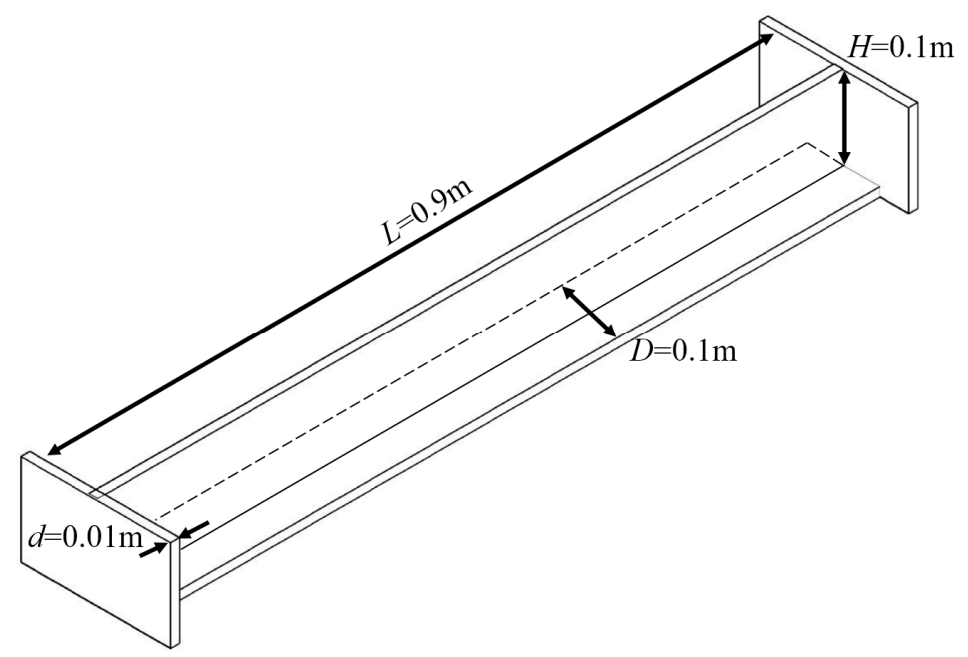

Figure 5. T-section prism size diagram.

In order to investigate the influence of the aspect ratio of the T-section prism on FIM responses and power generation characteristics, five T-section prisms with different $H$ values $(0.15 \mathrm{~m}, 0.12 \mathrm{~m}$, $0.1 \mathrm{~m}, 0.09 \mathrm{~m}$, and $0.08 \mathrm{~m}$ ) were tested in the experiments. The corresponding section aspect ratios $(\alpha=H / D)$ were $1.5,1.2,1.0,0.9$, and 0.8 , respectively. 


\subsection{Test Methods and Sensors}

\subsubsection{Displacement, Frequency, and Voltage}

The experimental study had two objectives. The first objective was to find the complete FIM responses of the T-section prism and to analyze the stability of each branch. The second aim was to estimate the energy conversion of the oscillation system in the complete FIM responses, and to investigate the influence of different aspect ratios on the power generation efficiency.

For oscillation characteristic tests, the main measurements were the displacements and frequencies of the prism. The displacements were tested by a magnetic induction displacement transducer with a direct current (DC) $24 \mathrm{~V}$ working voltage, which was supplied by external DC power. The testing range was $0-800 \mathrm{~mm}$, with a sensitivity of $0.1 \%$ and an error range of $\pm 0.05 \%$.

For the power generation tests, the connection method for the load resistances is presented in Figure 6. The minimum of the load resistances was $1 \Omega$, and the maximum was $50 \Omega$. In this study, 10 different load resistances were applied: $4 \Omega, 8 \Omega, 11 \Omega, 13 \Omega, 16 \Omega, 18 \Omega, 21 \Omega, 31 \Omega, 41 \Omega$, and $51 \Omega$. The experimental data was collected in the form of a voltage signal (shown in Figure 7) by the data acquisition system.

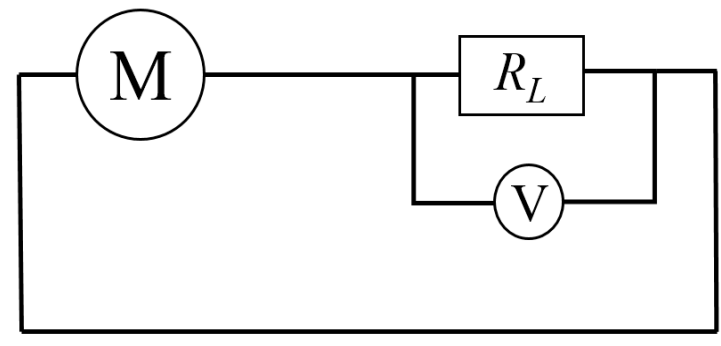

(a)

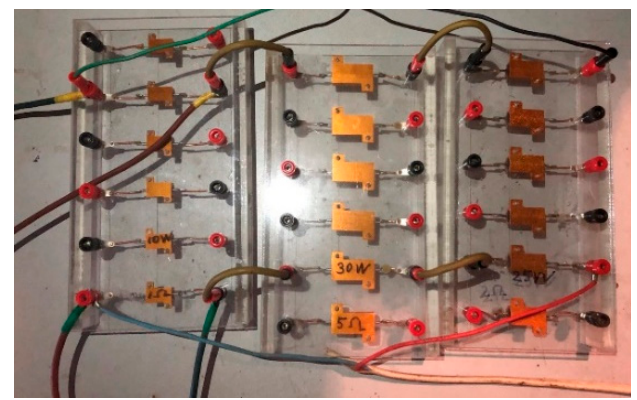

(b)

Figure 6. Voltage acquisition system: (a) contained circuit diagram; (b) connection resistances diagram.

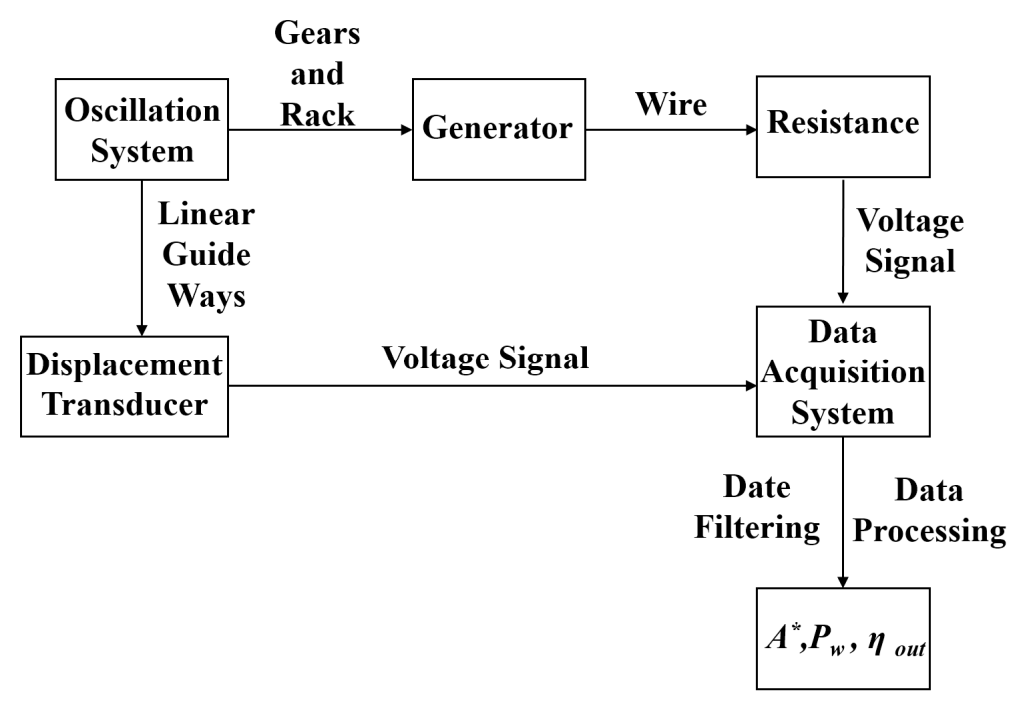

Figure 7. Test flow chart.

\subsubsection{Active Power and Power Generation Efficiency}

Active power $\left(P_{\text {harn }}\right)$ and power generation efficiency $\left(\eta_{\text {out }}\right)$ are the key parameters for evaluating

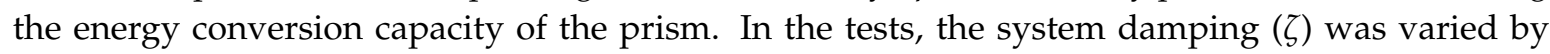


changing the load resistances $\left(R_{L}\right)$ [34]. The output voltage $(u)$ of the generator was measured by the data acquisition system, while $P_{\text {harn }}$ and $\eta_{\text {out }}$ of the system were calculated by the following equations: Instantaneous power expression: Equations (1)-(3)

$$
P(t)=\frac{u^{2}(t)}{R_{L}}
$$

where $P(t)$ is the instantaneous power, $u(t)$ is the instantaneous voltage, and $R_{L}$ is the load resistance.

The active power is written as:

$$
P_{\text {harn }}=\frac{1}{T} \int_{0}^{T} P(t) d t=\frac{1}{T} \int_{0}^{T} \frac{u^{2}(t)}{R_{L}} d t
$$

where $P_{\text {harn }}$ is the active power and $T$ is a period of oscillation.

The power generation efficiency is derived as:

$$
\eta_{\text {out }}=\frac{P_{\text {harn }}}{P_{w}}
$$

where $\eta_{\text {out }}$ is the power generation efficiency and $P_{w}$ is the total power in the fluid, which is written as:

$$
P_{w}=\frac{1}{2} \rho U^{3} D L
$$

where $\rho$ is the water density, $U$ is the incoming flow velocity, $D$ is the projection width of the T-section prism in the direction of the incoming flow, and $L$ is the prism length.

\subsection{Calibration of Stiffness and Damping}

A simple physical spring system designed by Xiang Yan et al. [36] was adopted to implement the stiffness of the system. First, free decay tests [35] with different spring stiffness values in the air were conducted to obtain the natural frequency $\left(f_{n}\right)$ of the experimental system.

The stiffness $(K)$ of the system varied from 800 to $1600 \mathrm{~N} / \mathrm{m}$. For each $K$, free decay tests were performed six times for the respective cases in air. $f_{n}$ was then calculated using a simple averaging method. The test results are shown in Table 1 . In these tests, the damping $\left(C_{\text {total }}\right)$ of the experimental

\begin{tabular}{|c|c|c|c|c|}
\hline$K(\mathrm{~N} / \mathrm{m})$ & $f_{n}(\mathrm{~Hz})$ & $m_{o s c}(\mathrm{~kg})$ & $C_{\text {total }}=C_{m}\left(\mathrm{~N} \cdot \mathrm{s} \cdot \mathrm{m}^{-1}\right)$ & $\zeta_{\text {total }}=\zeta_{m}$ \\
\hline 800 & 0.833 & 29.210 & 34.451 & 0.113 \\
\hline 1000 & 0.930 & 29.302 & 34.032 & 0.094 \\
\hline 1200 & 1.015 & 29.521 & 33.932 & 0.082 \\
\hline 1400 & 1.087 & 30.046 & 34.903 & 0.085 \\
\hline 1600 & 1.156 & 30.355 & 33.324 & 0.076 \\
\hline
\end{tabular}
system was equal to the mechanical damping $\left(C_{m}\right)$. The damping errors of different stiffnesses were within the allowable ranges of the tests (the average was $\pm 5 \%$ ).

Table 1. Free decay test results by varying stiffnesses.

The damping ratio $\left(\zeta_{\text {total }}\right)$ of the test results could be determined by using the logarithmic decrement method, which is expressed as:

$$
\zeta_{\text {total }}=\frac{1}{2 \pi} \ln \left(\frac{A_{i}}{A_{i+1}}\right)
$$

where $A_{i}$ is the amplitude of the $i$ th peak and $\zeta_{\text {total }}$ is the damping ratio of the experimental system. 
The $C_{\text {total }}$ of the oscillation system can be calculated as:

$$
C_{\text {total }}=2 \zeta \sqrt{m_{o s c} K}
$$

where $K$ is the system stiffness, $C_{\text {total }}$ is the damping of the oscillation system, and $m_{o s c}$ is the system mass.

The FIM responses of the T-section prism with different $\zeta_{\text {total }}$ are described in this section. The $\zeta_{\text {total }}$ of the system is varied by changing $R_{L}$, and the parameters are shown in Table 2.

Table 2. Free decay test results by varying load resistances.

\begin{tabular}{cccccc}
\hline $\boldsymbol{R}_{\boldsymbol{L}}(\boldsymbol{\Omega})$ & $f_{\boldsymbol{n}} \mathbf{( H z )}$ & $\boldsymbol{m}_{\text {osc }} \mathbf{( k g )}$ & $\boldsymbol{C}_{\text {total }} \mathbf{( \mathbf { N } \cdot \mathbf { s } \cdot \mathbf { m } ^ { - \mathbf { 1 } } )}$ & $\boldsymbol{C}_{\text {harn }} \mathbf{( \mathbf { N } \cdot \mathbf { s } \cdot \mathbf { m } ^ { - \mathbf { 1 } } )}$ & $\zeta_{\text {total }}$ \\
\hline 4.210 & 1.084 & 30.046 & 142.212 & 107.312 & 0.347 \\
8.130 & 1.089 & 30.046 & 125.192 & 90.292 & 0.305 \\
11.250 & 1.086 & 30.046 & 100.951 & 66.051 & 0.246 \\
13.410 & 1.087 & 30.046 & 85.482 & 50.582 & 0.208 \\
16.330 & 1.082 & 30.046 & 72.783 & 37.883 & 0.177 \\
18.250 & 1.086 & 30.046 & 67.547 & 32.647 & 0.165 \\
21.450 & 1.088 & 30.046 & 62.842 & 27.942 & 0.153 \\
31.540 & 1.090 & 30.046 & 52.115 & 17.215 & 0.127 \\
41.110 & 1.086 & 30.046 & 48.680 & 13.780 & 0.119 \\
51.620 & 1.084 & 30.046 & 46.974 & 12.074 & 0.115 \\
$\infty$ & 1.087 & 30.046 & 34.903 & 0.000 & 0.085 \\
\hline
\end{tabular}

\section{Results and Discussion}

\subsection{Vibration Characteristic Analysis}

\subsubsection{Amplitudes and Frequencies}

To explore the FIM performances of the T-section prism in water, a series of FIM tests with 10 different $R_{L}$ were carried out at $K=1400 \mathrm{~N} / \mathrm{m}$ (as seen in Section 2.4). In Figure 8a, $A^{*}$ is the amplitude ratio $\left(A^{*}=A / D\right), A$ denotes the average amplitude of continuous oscillation for $30 \mathrm{~s}, D$ is the projection width of the T-section prism in the direction of the incoming flow, $U_{r}$ is the reduced velocity $\left(U_{r}=U /\left(D \cdot f_{n}\right)\right), U$ is the incoming flow velocity, and $f_{n}$ is the natural frequency in air. In Figure $8 \mathrm{~b}$, $f^{*}$ is the frequency ratio $\left(f^{*}=f_{o s c} / f_{n}\right)$, and $f_{o s c}$ is the main frequency of oscillation obtained from the displacement time-history curves by the Fast Fourier Transformation (FFT) method. $A^{*}$ and $f^{*}$ vary with $U_{r} ; U$ and the Reynolds number $R e$ for all $R_{L}$ cases are plotted in Figures 8-11, respectively.

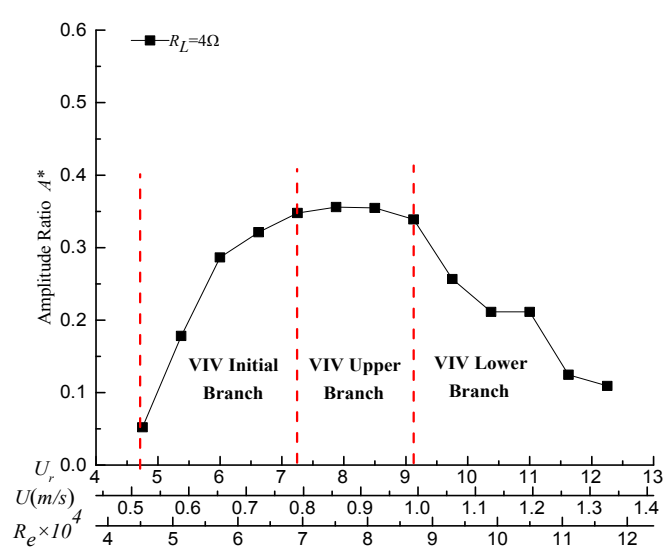

(a)

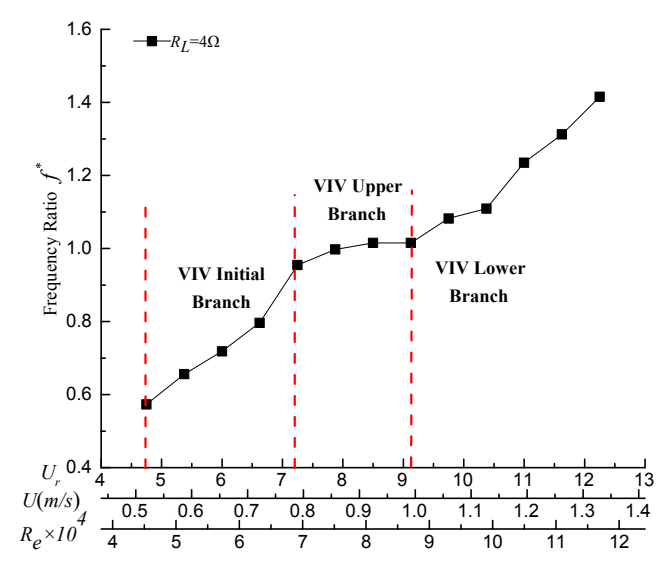

(b)

Figure 8. Vortex-induced vibration (VIV) oscillation characteristics $\left(R_{L}=4 \Omega, \zeta=0.347\right)$ : (a) response of the amplitude ratio; (b) response of the frequency ratio. 


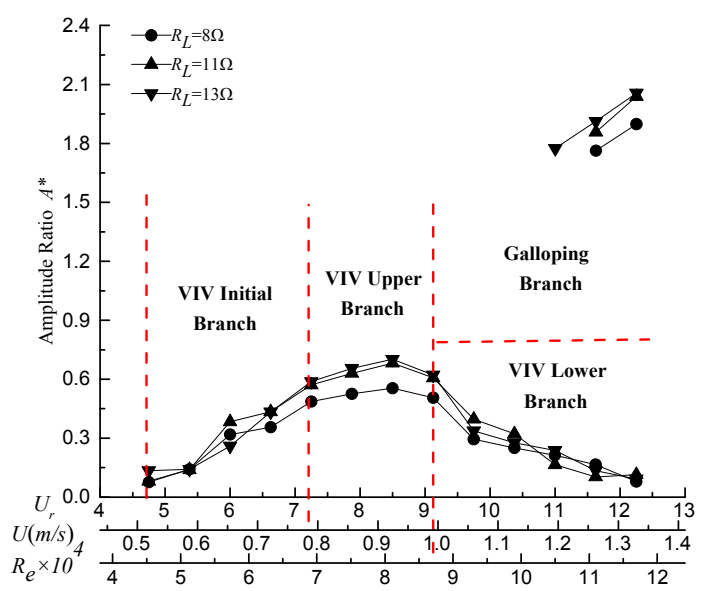

(a)

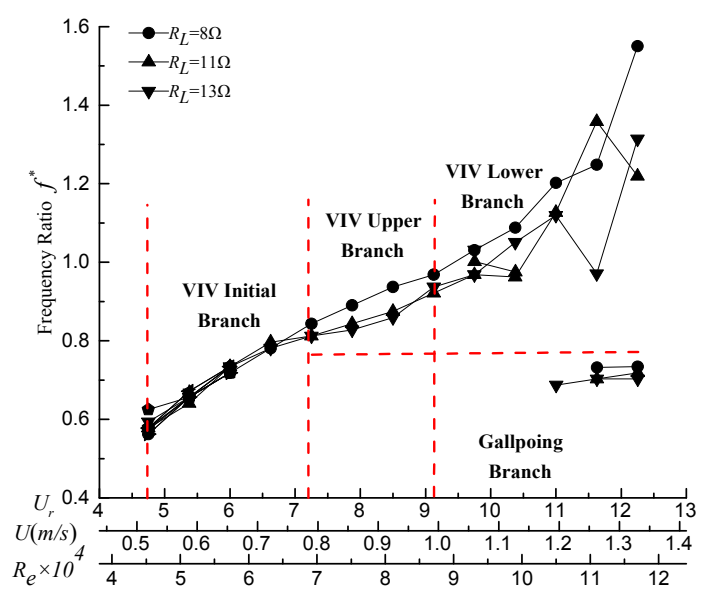

(b)

Figure 9. High galloping (HG) oscillation characteristics $8 \Omega \leq R_{L} \leq 13 \Omega(0.208 \leq \zeta \leq 0.305)$ : (a) response of the amplitude ratio; (b) response of the frequency ratio.

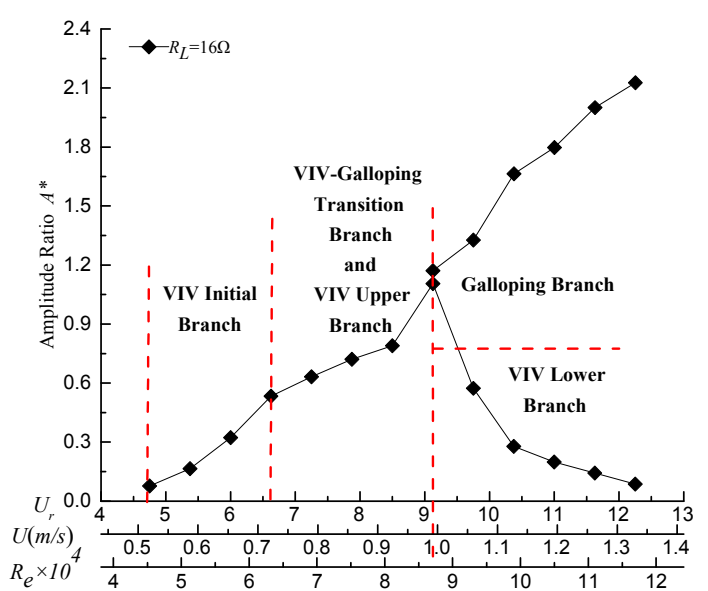

(a)

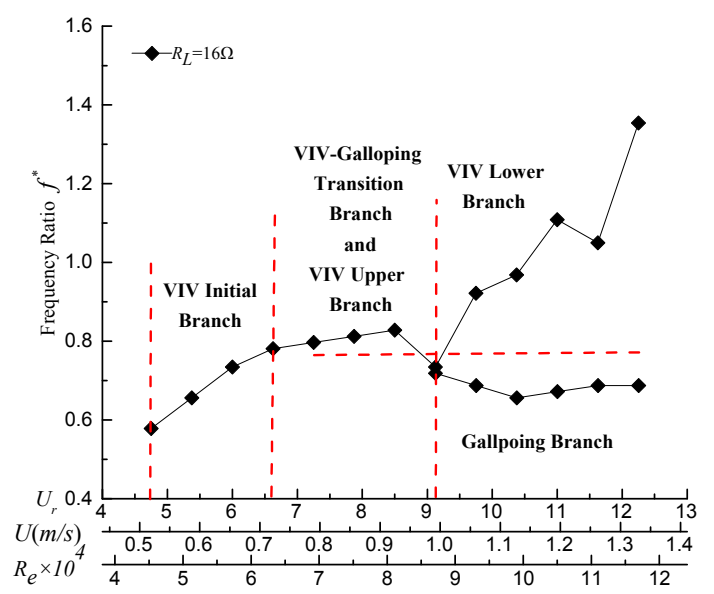

(b)

Figure 10. Critical galloping (CG) oscillation characteristics $R_{L}=16 \Omega(\zeta=0.177)$ : (a) response of the amplitude ratio; $(\mathbf{b})$ response of the frequency ratio.

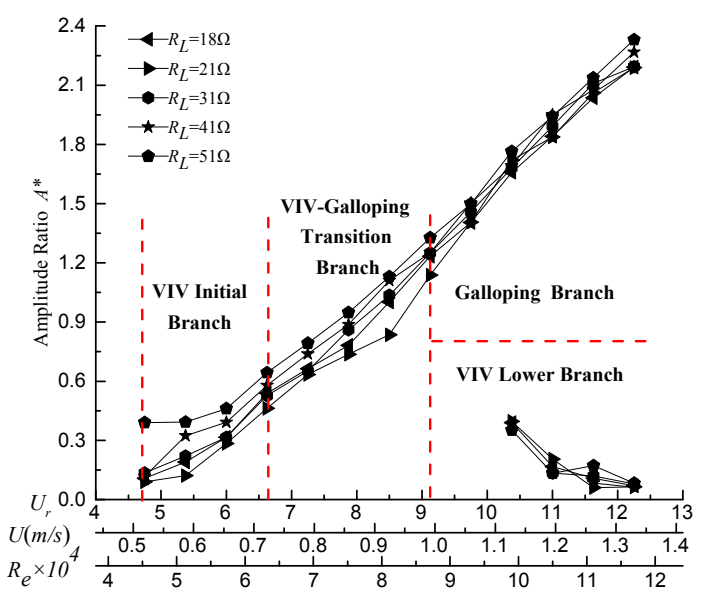

(a)

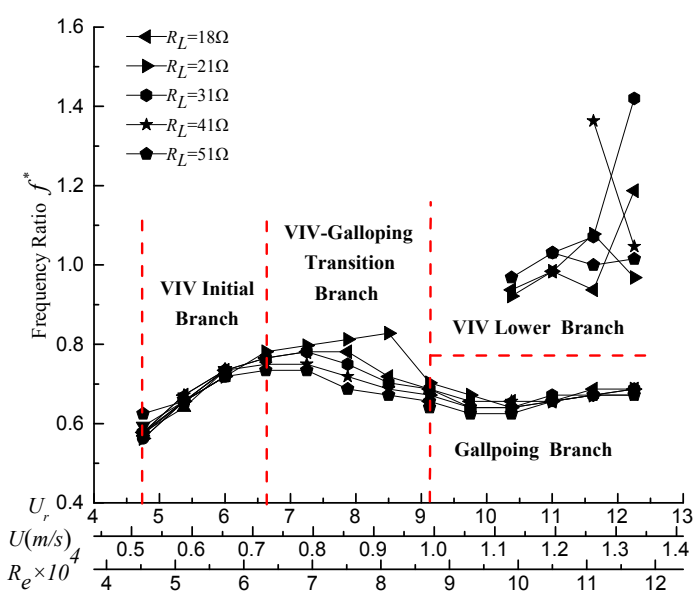

(b)

Figure 11. Soft galloping (SG) oscillation characteristics $18 \Omega \leq R_{L} \leq 51 \Omega(0.115 \leq \zeta \leq 0.165)$ :

(a) response of the amplitude ratio; (b) response of the frequency ratio. 
(1) VIV: For $R_{L}=4 \Omega(\zeta=0.347)$, the T-section prism was only characterized by the typical VIV in the range of $4.75 \leq U_{r} \leq 12.25$ (shown in Figure 8), and the prism did not present any signs of galloping. For $4.75 \leq U_{r} \leq 7.25, A^{*}$ and $f^{*}$ were small but increased rapidly, indicating that the prism underwent the VIV initial branch. The motion of the prism was induced by vortex shedding, but the oscillation and vortex shedding were not synchronized well. For $7.25 \leq U_{r} \leq 9.125, A^{*}$ stayed at 0.35 , and $f^{*}$ increased sharply and was maintained at approximately 1 . The oscillation entered the VIV upper branch (lock-in range), which was more stable than the VIV initial branch. For $U_{r} \geq 9.125$, $A^{*}$ rapidly collapsed down to about 0.1 , and $f^{*}$ increased rapidly to a high level $\left(f^{*}>>1\right)$. In this range, the oscillation and vortex shedding were not synchronized well, demonstrating that the prism underwent the VIV lower branch.

(2) HG: For $8 \Omega \leq R_{L} \leq 13 \Omega(0.208 \leq \zeta \leq 0.305)$, two modes of motion could be observed in the response of the prism (shown in Figure 9). If the prism oscillated freely (without any external forces acting on the prism), the prism underwent complete VIV responses as $U_{r}$ increased or decreased in the range of $4.75 \leq U_{r} \leq 12.25$. However, when $U_{r}$ decreased from 12.25 and the prism was manually pushed by a threshold initial displacement (exceeding $1 \times \mathrm{D}$ ), the prism first entered the galloping branch, accompanied by a large amplitude $\left(A^{*} \approx 2.3\right)$ and a stable frequency $\left(f^{*}=0.7\right)$. As $U_{r}$ decreased, $A^{*}$ decreased gradually and the oscillation was still in the galloping branch. When $U_{r}$ reached 11-11.625, the oscillation was suddenly suppressed, and the oscillation mode transformed from galloping to VIV (lower branch), accompanied by a sudden collapse in $A^{*}$ from 1.8 to 0.2 , and a sudden jump in $f^{*}$ from 0.7 to 1 . It was concluded that the prism cannot be self-excited from VIV to galloping in any case, without any external conditions (such as a threshold initial displacement) or forces acting on the prism, no matter whether the velocity decreases or increases, demonstrating that the prism experiences HG responses in these cases.

(3) Critical galloping (CG): For $R_{L}=16 \Omega(\zeta=0.177)$, the system presented both complete VIV responses and galloping while the two branches intersected (shown in Figure 10). When $U_{r}$ increased from 4.75 to 12.25 , the prism experienced a VIV initial branch, a VIV upper branch, and a VIV lower branch. If a threshold initial displacement was applied at $U_{r}=12.25$, the oscillation was suddenly enhanced, and the oscillation mode dramatically transformed from VIV (initial branch) to galloping, accompanied by a sudden jump in $A^{*}$ from 0.08 to 2.12 as well as a sudden collapse in $f^{*}$ from 1.35 to 0.68 . Afterwards, the oscillation entered the galloping branch. $A^{*}$ gradually decreased, with the decrease of $U_{r}$, and $f^{*}$ was maintained at about 0.7 . No oscillation suppression phenomena occurred until the oscillation underwent the VIV upper branch where the intersection point was $U_{r}=9.125$. However, at $U_{r} \geq 9.125$, galloping could be induced by external excitation. This condition is actually a critical oscillation mode between HG and SG, which can be referred to as "critical galloping" (CG).

(4) SG. For $18 \Omega \leq R_{L} \leq 51 \Omega(0.115 \leq \zeta \leq 0.165)$, two modes of motion were observed in the responses of the prism. If the prism oscillated freely (without any external forces acting on the prism), the T-section prism experienced the VIV initial branch, followed by the VIV-galloping transition branch, and ended with the galloping branch (shown in Figure 11). In the range of $4.75 \leq U_{r} \leq 6.625, A^{*}$ and $f^{*}$ were small but increased rapidly, indicating that the prism underwent the VIV initial branch. In the range of $6.625 \leq U_{r} \leq 9.125, A^{*}$ continued to grow with a strong uptrend to 1.5 , while $f^{*}$ decreased to about 0.7 with a slight downward trend. For $U_{r} \geq 9.75, A^{*}$ continued to increase up to 2.36 , while $f^{*}$ almost remained at 0.7 . The oscillation underwent the fully developed galloping branch. On the other hand, at $U_{r}=12.25$, external suppression was applied to the prism; thus, the oscillation presented a low $A^{*} \approx 0.2$ and a high $f^{*} \approx 1.4$, the system experienced the VIV lower branch until $U_{r} \leq 10.375$, and the oscillation mode returned to galloping. For $6.625 \leq U_{r} \leq 10.375$, the oscillation was self-excited from VIV to galloping (regardless of whether the velocity increased or decreased). If $U_{r}$ continued to increase, the oscillation would be maintained within the range of galloping. For $U_{r} \geq 10.375$, if there was an external suppression, the oscillation would be converted to the VIV lower branch and could not be self-excited to galloping. 
It can be concluded that the oscillation responses of the T-section prism were similar to those of the triangular prism and the PTC circular cylinder. The occurrence conditions of different FIM branches of the T-section prism are listed in Table 3.

Table 3. VIV and galloping occurrence conditions with $K=1400 \mathrm{~N} / \mathrm{m}$.

\begin{tabular}{cccccc}
\hline Oscillation Form & $\boldsymbol{R}_{\boldsymbol{L}}$ & $\boldsymbol{U}_{\boldsymbol{r}}$ & External Excitation & External Suppression & Self-Excited by VIV \\
\hline VIV & $4 \Omega$ & $4.75 \sim 12.25$ & & & $\sqrt{ }$ \\
HG & $8 \sim 13 \Omega$ & $11 \sim 12.25$ & $\sqrt{ }(\mathrm{HG})$ & & \\
CG & $16 \Omega$ & $9.125 \sim 12.25$ & & $\sqrt{ }$ (VIV Low B) & $\sqrt{ }$ \\
SG & $18 \sim 51 \Omega$ & $10.375 \sim 12.25$ & & $\sqrt{ }$ (VIV Low B) & $\sqrt{ }$ \\
\hline
\end{tabular}

\subsubsection{Time-History and Frequency Spectrum of Each Branch}

In order to explore the oscillation characteristics of each branch, four typical time-history curves and frequency spectra are depicted in this section (plotted in Figures 12 and 13). The physical parameters of the oscillation system are listed in Table 4.

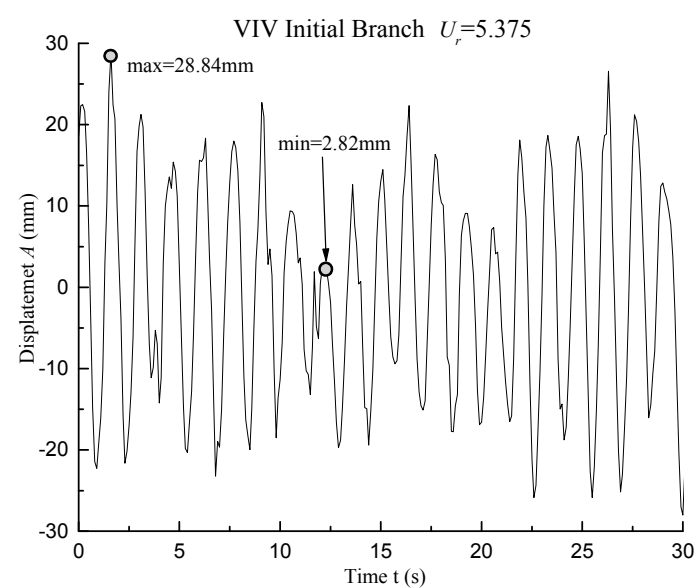

(a)

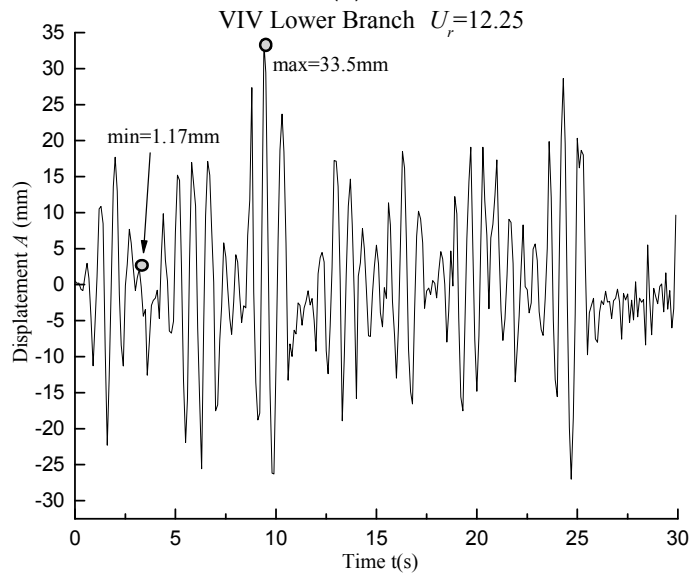

(c)

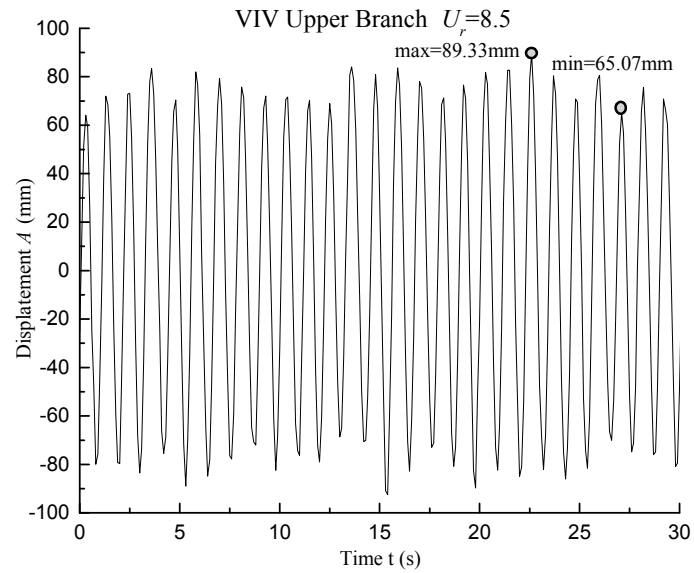

(b)

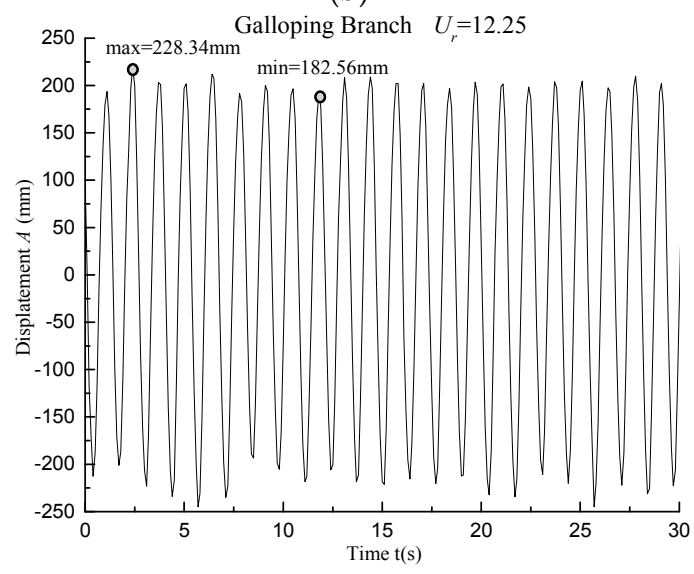

(d)

Figure 12. Typical flow-induced motion displacement time-history curves: (a) VIV initial branch; (b) VIV upper branch; (c) VIV lower branch; (d) galloping branch. 


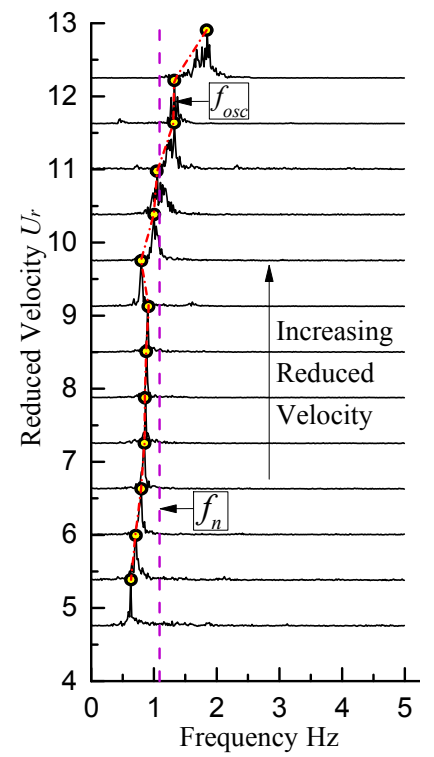

(a)

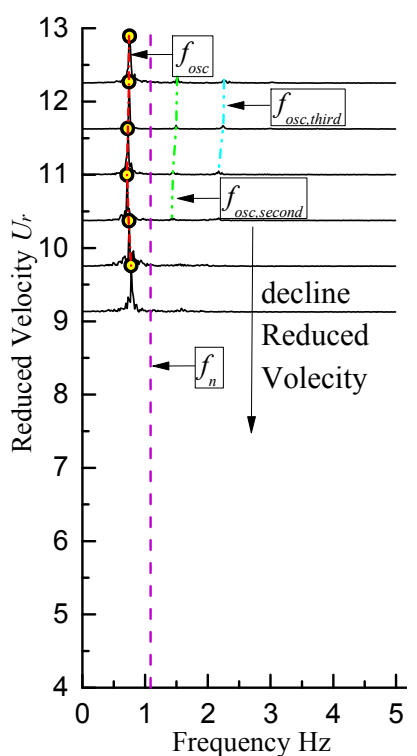

(b)

Figure 13. Typical oscillation frequency spectrum: (a) complete VIV branch; (b) galloping branch.

Table 4. Oscillation form and parameters list.

\begin{tabular}{ccccc}
\hline $\boldsymbol{R}_{\boldsymbol{L}}(\boldsymbol{\Omega})$ & $C_{\text {total }}\left(\mathbf{N} \cdot \mathbf{s} \cdot \mathbf{m}^{-\mathbf{1}}\right)$ & $C_{\text {harn }} \mathbf{( N \cdot \mathbf { s } \cdot \mathbf { m } ^ { - \mathbf { 1 } } )}$ & $\zeta$ & Oscillation Form \\
\hline 16 & 72.783 & 37.883 & 0.177 & CG \\
\hline
\end{tabular}

\section{(1) Time-History}

For $U_{r}=5.375$, the prism underwent the VIV initial branch. The time-history curve of the displacement is shown in Figure 12a. It was observed that the minimum of the positive displacement was $2.82 \mathrm{~mm}$, the maximum was $28.84 \mathrm{~mm}$, and the difference between the maximum and minimum was $1.25 A(A=16 \mathrm{~mm})$, indicating that the amplitude was low and the oscillation was unstable.

As $U_{r}$ reached 8.5, the oscillation mode entered the VIV upper branch, as shown in Figure 12b. It was observed that the minimum of the positive displacement was $65.07 \mathrm{~mm}$, the maximum was $89.33 \mathrm{~mm}$, and the difference between the maximum and minimum was $0.31 A(A=78 \mathrm{~mm})$. Moreover, the prism underwent a more stable oscillation, although there was a certain fluctuation response amplitude.

For $U_{r}=12.25$, it was observed that the minimum of the positive displacement was $1.17 \mathrm{~mm}$, the maximum was $33.50 \mathrm{~mm}$, and the difference between the maximum and minimum was $4.04 \mathrm{~A}$ $(A=8 \mathrm{~mm})$. The oscillation amplitude fluctuation was large and the performance was very unstable, as shown in Figure 12c, demonstrating that the prism underwent the VIV lower branch.

For $U_{r}=12.25$ (galloping branch), the minimum of the positive displacement was $182.56 \mathrm{~mm}$, the maximum was $228.34 \mathrm{~mm}$, and the difference between the maximum and minimum was $0.21 \mathrm{~A}$ ( $A=212 \mathrm{~mm}$ ), indicating that the oscillation amplitude was large and the fluctuation was small, as shown in Figure 12d.

Both stability and amplitude are key parameters for energy harvesting. Thus, the galloping branch is the most suitable branch for energy extraction and utilization.

(2) Frequency Spectrum

The frequency spectrum is important for describing the oscillation characteristics and mechanical energy. The oscillation frequency of the prism was extracted by using the FFT method from the displacement time-history curves (shown in Figure 13). Figure 13a plots the frequency spectrum of the complete VIV branch and Figure 13b plots the frequency spectrum of the galloping branch, while the trends of $f_{n}$ and $f_{o s c}$ are marked by dashed lines. 
For $4.75 \leq U_{r} \leq 6.625$, the prism underwent the VIV initial branch, and the frequency band was wider and exhibited double peaks in the frequency spectrum, revealing that periodicity of oscillation and mechanical energy was poor.

In the range of $6.625 \leq U_{r} \leq 9.125$, the prism entered the VIV upper branch, the frequency band became narrower, and the main frequency was clearly observed. The periodicity of oscillation was significantly enhanced, the prism oscillated more stably, and the main frequency was concentrated at $0.88 \mathrm{~Hz}$.

For $U_{r} \geq 9.125$, two modes of oscillation were observed. When the prism oscillated freely (without external excitation), the prism went into the VIV lower branch, the main frequency was not obvious, the frequency band widened, and the energy was very dispersed.

On the other hand, if the prism was manually pushed by a threshold initial displacement (exceeding $1 \times \mathrm{D}$ ), the prism entered the galloping branch with a narrowed frequency band and evident first dominant frequency, as shown in Figure 13b. It was noted that in the galloping branch, the oscillation was mainly caused by the instability of the lift force due to the T-section prism with sharp sections. Because of the lower energy and the higher frequency of the vortex shedding, the effect of vortex shedding on the prism was weaker, resulting in the second and third dominant frequencies being hardly observed in the frequency spectrum.

The conclusions of the time-history curves and frequency spectra are as follows: (1) different fluctuations are observed in each branch-the VIV upper branch and the galloping branch have higher amplitudes and more stable oscillations; (2) the VIV upper branch and the galloping branch have a better periodicity and frequency spectrum. In summary, the VIV upper branch and the galloping branch are more suitable for energy harvesting, but the galloping branch is better (shown in Table 5).

Table 5. Oscillation characteristics of different branches.

\begin{tabular}{ccccc}
\hline Branch & VIV Initial & VIV Upper & VIV Lower & Galloping \\
\hline Amplitude & low & high & low & highest \\
Stability & bad & good & bad & best \\
Frequency & bad & good & bad & best \\
\hline
\end{tabular}

\subsubsection{Summary}

The specific findings are listed as follows:

(1) With an increase of damping (decrease in load resistances), the T-section prism oscillation mode gradually changes from SG $\left(0.115 \leq \zeta \leq 0.165,18 \Omega \leq R_{L} \leq 51 \Omega\right)$ to $C G\left(\zeta=0.177, R_{L}=16 \Omega\right)$, and eventually to $H G\left(0.208 \leq \zeta \leq 0.305,8 \Omega \leq R_{L} \leq 13 \Omega\right)$. The oscillation mode of SG, HG, and CG all contain VIV branches and galloping branches, the only difference being whether these can be self-excited from VIV to galloping.

(2) The analysis of the displacement time-history curves and the frequency spectra demonstrates that the stability and intensity of the VIV upper branch and the galloping branch are both better-performing, and that the maximum amplitude of the galloping branch is larger. It can be concluded that at a high velocity $\left(U_{r} \geq 10.375, U \geq 1.128 \mathrm{~m} / \mathrm{s}\right)$ the galloping branch is better for harvesting energy, and at a lower velocity $\left(6.625 \leq U_{r} \leq 9.125,0.720 \leq U \leq 0.992 \mathrm{~m} / \mathrm{s}\right)$ the VIV upper branch is better. Power generation with different load resistances will be presented in the next section.

\subsection{Power Generation Analysis}

\subsubsection{Active Power Analysis}

The variations of active power $P_{\text {harn }}$ of the generator versus incoming flow velocity $U$, reduced flow $U_{r}$, and Reynolds number $R e$ are plotted in Figure 14. $P_{\text {harn }}$ is the average of the instantaneous power under continuous oscillation for $30 \mathrm{~s}$. For $U_{r} \geq 6$, the oscillation system started to output 
electrical energy and $P_{\text {harn }}$ was smaller, as the system experienced the complete VIV responses and the $P_{\text {harn }}$ did not exceed $5 \mathrm{~W}$. As galloping occurred, $P_{\text {harn }}$ increased to over $20 \mathrm{~W}$. The details are described as follows:

(1) For $18 \Omega \leq R_{L} \leq 51 \Omega$, SG occurred.

As $U_{r}$ increased, the prism experienced the VIV initial branch, the VIV-galloping transition branch, and the galloping branch. $P_{\text {harn }}$ monotonically increased with $U_{r}$ and the maximum $P_{\text {harn }}$ appeared at the galloping branch $\left(P_{\text {harn, } \max }=13 \mathrm{~W}, R_{L}=18 \Omega, U_{r}=12.25\right)$. It was noted that the higher the $U_{r}$, the lower the $R_{L}$, and the more energy was converted.

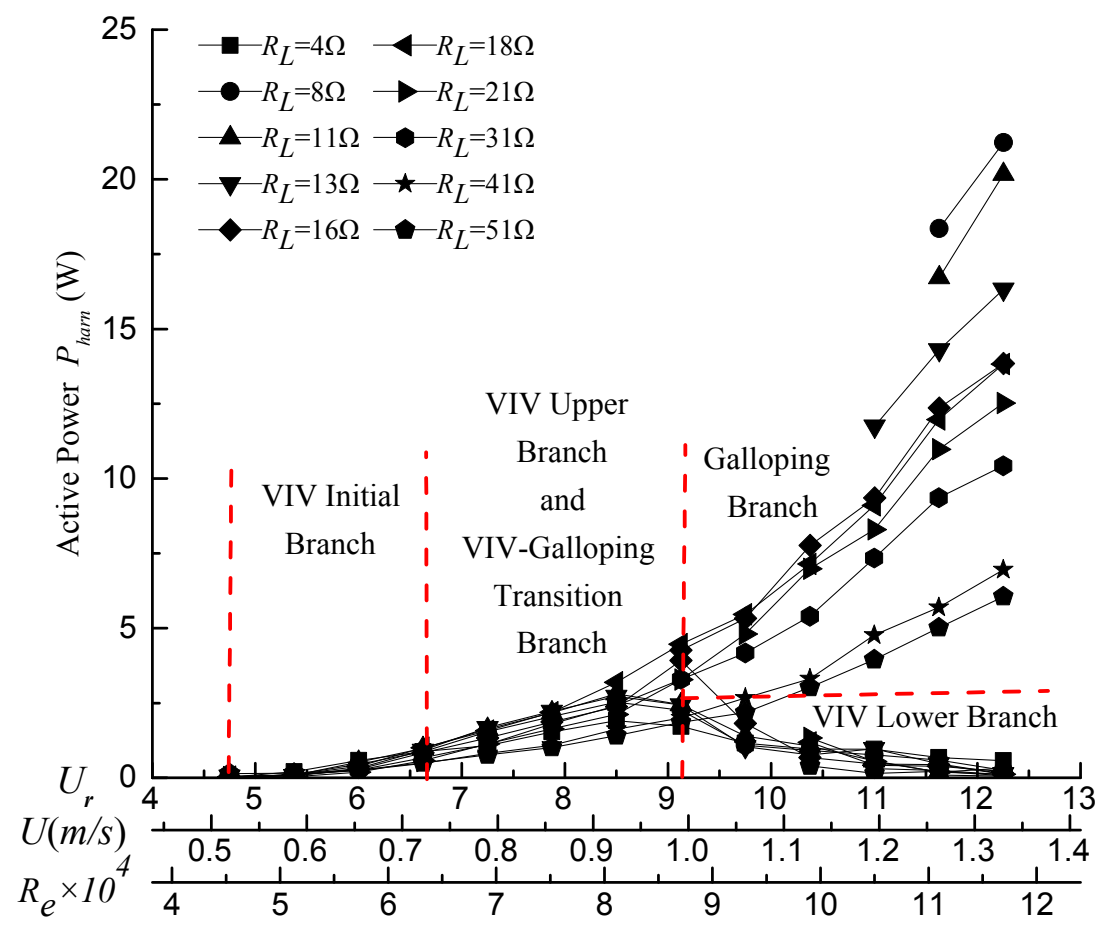

Figure 14. T-section prism power generation active power.

(2) For $8 \Omega \leq R_{L} \leq 16 \Omega$, HG occurred, and two oscillation modes were observed.

(i) Without external excitation (such as forces, threshold initial displacement, etc.)

The prism only experienced VIV branches. $P_{\text {harn }}$ rose with the increase of $U_{r}$ at the VIV initial branch and at the VIV upper branch $\left(P_{\text {harn, } \max }=3.92 \mathrm{~W}, R_{L}=16 \Omega, U_{r}=9.125\right)$. Afterwards, $P_{\text {harn }}$ decreased to lower than $1 \mathrm{~W}$, as $U_{r}$ rose up to 12.25 in the VIV lower branch.

(ii) An external excitation was applied to the prism.

At $U_{r}=12.25$, if the threshold initial displacement was applied to the prism, the oscillation directly jumped into the galloping branch. As $U_{r}$ decreased, $P_{\text {harn }}$ dropped rapidly. In the present tests, the maximum $P_{\text {harn }}$ was $21.23 \mathrm{~W}\left(U=1.332 \mathrm{~m} / \mathrm{s}, R e=116396, R_{L}=8 \Omega\right)$. The maximum $P_{\text {harn }}$ was close and it was slightly lower than that of the PTC circular cylinder $\left(P_{\text {harn }, \max }=23.54 \mathrm{~W}\right)$, as reported by Lin, D [44].

(3) For $R_{L}=4 \Omega$, only VIV branches were observed.

The variation of $P_{\text {harn }}$ versus $U_{r}$ for $R_{L}=4 \Omega$ was similar to those for $8 \Omega \leq R_{L} \leq 16 \Omega$ in VIV branches. Within the test flow velocity range, the prism did not experience any forms of galloping. The maximum $P_{\text {harn }}$ was $2.5 \mathrm{~W}$ at $U_{r}=8.5$ (VIV upper branch).

\subsubsection{Efficiency Analysis}

The variations of efficiency $\eta_{\text {out }}$ versus incoming flow velocity $U$, reduced flow $U_{r}$, and Reynolds number $R e$ are plotted in Figure 15. $\eta_{\text {out }}$ was calculated using Equation (3) (shown in Section 2.3). 


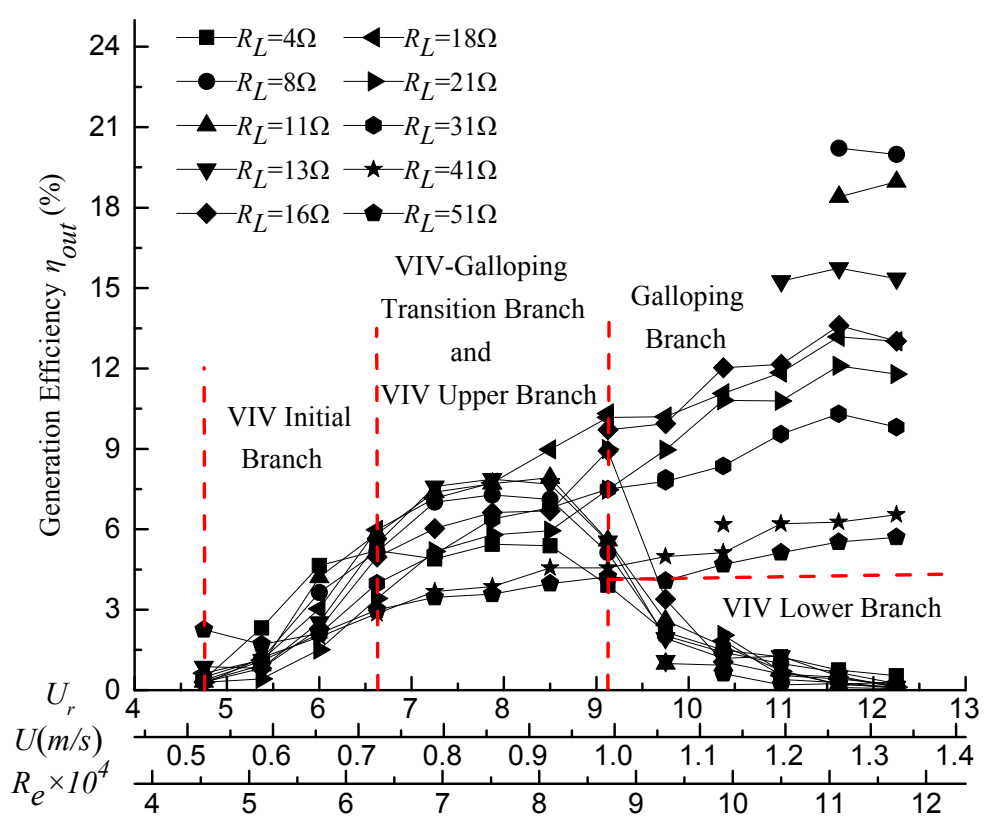

Figure 15. T-section prism power generation efficiency.

It was observed that $\eta_{\text {out }}$ did not exceed 9\% in VIV branches (whether in SG or HG responses). The corresponding load resistance was $R_{L}=16 \Omega$ and the corresponding velocity was $U_{r}=9.125$ $(U=0.992 \mathrm{~m} / \mathrm{s}, R e=86703)$. On the contrary, the maximum $\eta_{\text {out }}$ reached $20.2 \%$ in the galloping branch, which was close to the test results of the smooth (22\%) and the PTC $(28 \%)$ circular cylinders. The corresponding load resistance was $R_{L}=8 \Omega$, and the corresponding velocity was $U_{r}=11.625$ $(U=1.264 \mathrm{~m} / \mathrm{s}, R e=110,458)$. It is concluded that the energy conversion efficiency in the galloping branch is much higher than that of the VIV branches for the T-section prism.

In addition, in most of the galloping branches, especially for small load resistances $\left(R_{L}<31\right)$ or high damping ratios $(\zeta>0.127)$, a slight declining trend was observed for $U_{r}$ exceeding 11.625 in the present tests. It was indicated that $U_{r}=11.625$ was the optimal velocity.

\subsubsection{Stability Analysis}

In order to describe the power generation stability of each oscillation branch, the instantaneous voltage time-history curves and instantaneous power time-history curves of four oscillation branches are discussed in this section (shown in Figures 16 and 17).

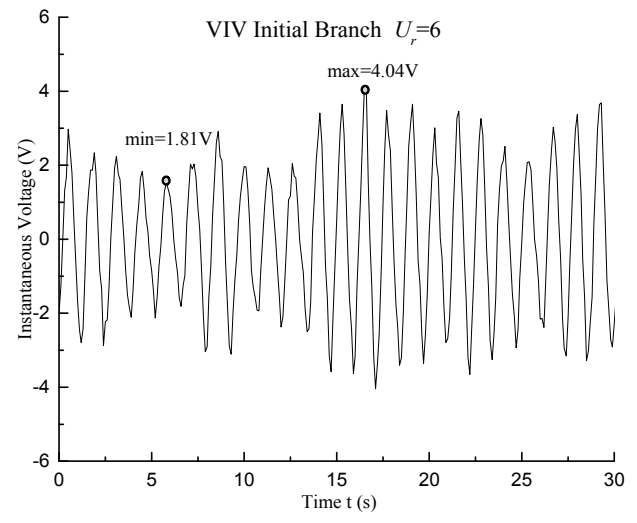

(a)

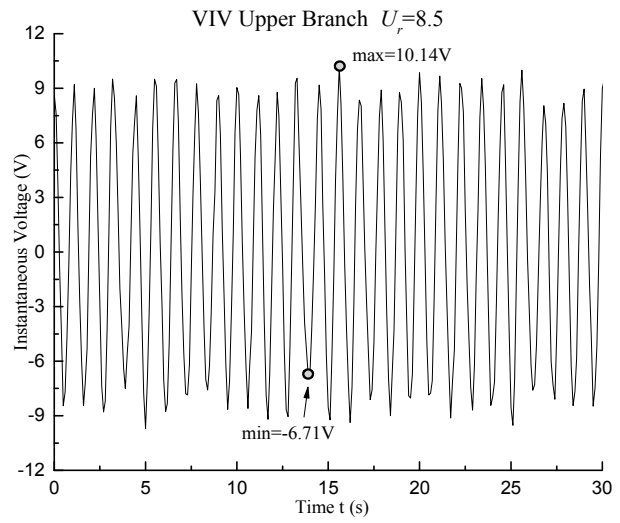

(b)

Figure 16. Cont. 


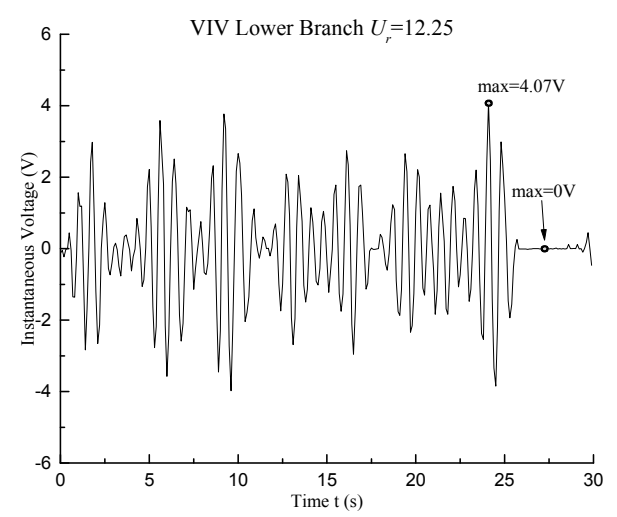

(c)

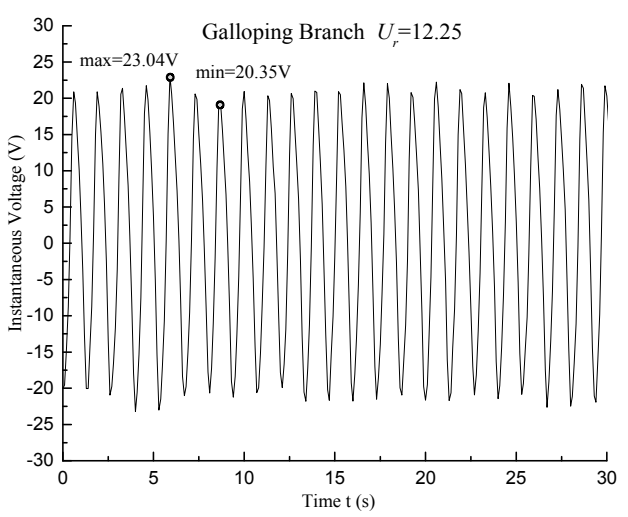

(d)

Figure 16. Instantaneous voltage time-history curves of T-section prism power generation with $R_{L}=16 \Omega$ : (a) VIV initial branch; (b) VIV upper branch; (c) VIV lower branch; (d) galloping branch.

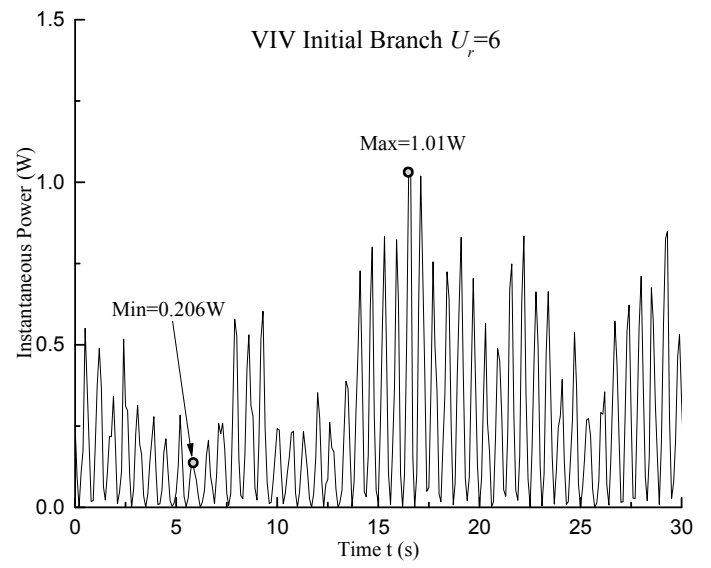

(a)

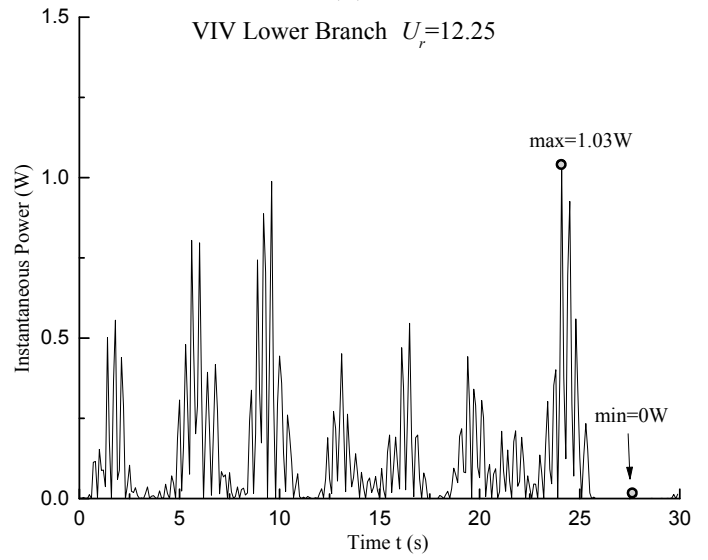

(c)

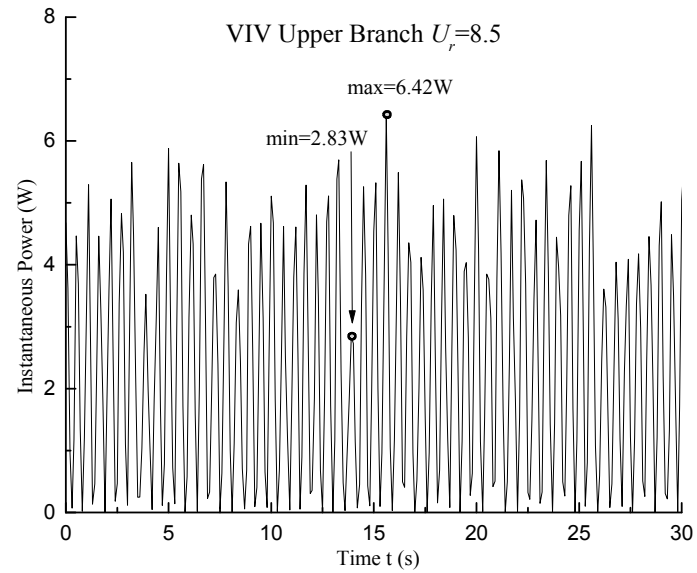

(b)

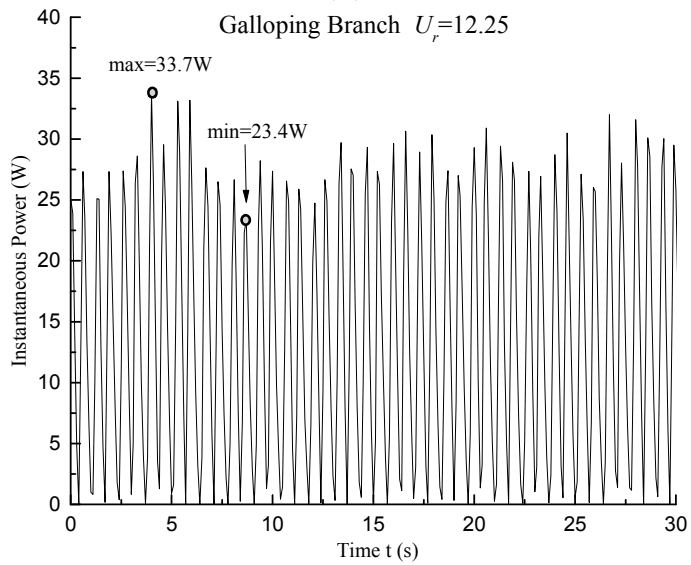

(d)

Figure 17. Power generation instantaneous power time-history curves of the T-section prism with $R_{L}=16 \Omega$ : (a) VIV initial branch; (b) VIV upper branch; (c) VIV lower branch; (d) galloping branch.

Figure 16a,c show that the instantaneous voltage peaks fluctuated greatly when the prism underwent the VIV initial branch $\left(V_{\max }=4.04 \mathrm{~V}, V_{\min }=1.81 \mathrm{~V}\right)$ and the VIV lower branch $\left(V_{\max }=4.07 \mathrm{~V}, V_{\min }=0 \mathrm{~V}\right)$. This led directly to the instability of the instantaneous power of the VIV initial branch $\left(P_{\max }=1.01 \mathrm{~W}, P_{\min }=0.206 \mathrm{~W}\right)$ and the VIV lower branch $\left(P_{\max }=1.03 \mathrm{~W}, P_{\min }=0 \mathrm{~W}\right)$, as shown in Figure 17a,c. Additionally, the instantaneous voltages of the VIV initial branch and the 
VIV lower branch were small, so that the powers were not as high as the instantaneous active power in the VIV upper branch $\left(P_{\max }=6.42 \mathrm{~W}, P_{\min }=2.83 \mathrm{~W}\right)$ and in the galloping branch $\left(P_{\max }=33.7 \mathrm{~W}\right.$, $\left.P_{\min }=23.4 \mathrm{~W}\right)$, as shown in Figure 17b,d. The peak instantaneous voltages of the VIV upper branch $\left(V_{\max }=10.14 \mathrm{~V}, V_{\min }=-6.71 \mathrm{~V}\right)$ and the galloping branch $\left(V_{\max }=23.04 \mathrm{~V}, V_{\min }=20.35 \mathrm{~V}\right)$ had small fluctuations, as shown in Figure 16b,d. The reason for this was that the two branches had better oscillation stability. In addition, the instantaneous power of the galloping branch was much higher and the quality of the output energy was better.

In order to further discuss the stability of the harnessed power, the difference coefficient $C_{v}$ was introduced as follows:

$$
C_{v}=\frac{\sigma_{p}}{P_{\text {harn,max }}}
$$

where $\sigma_{p}$ is the square deviation of all peak powers, and $P_{\text {harn,max }}$ is the average of all peak powers. It was noted that a higher $C_{v}$ corresponded to a more unstable power output; otherwise, it corresponded to a more stable power output. The power generation difference coefficient $C_{v}$ was calculated based on Equation (6), and is shown in Figure 18. In different branches, the stability of the oscillation was quite different. The performances are described as follows:

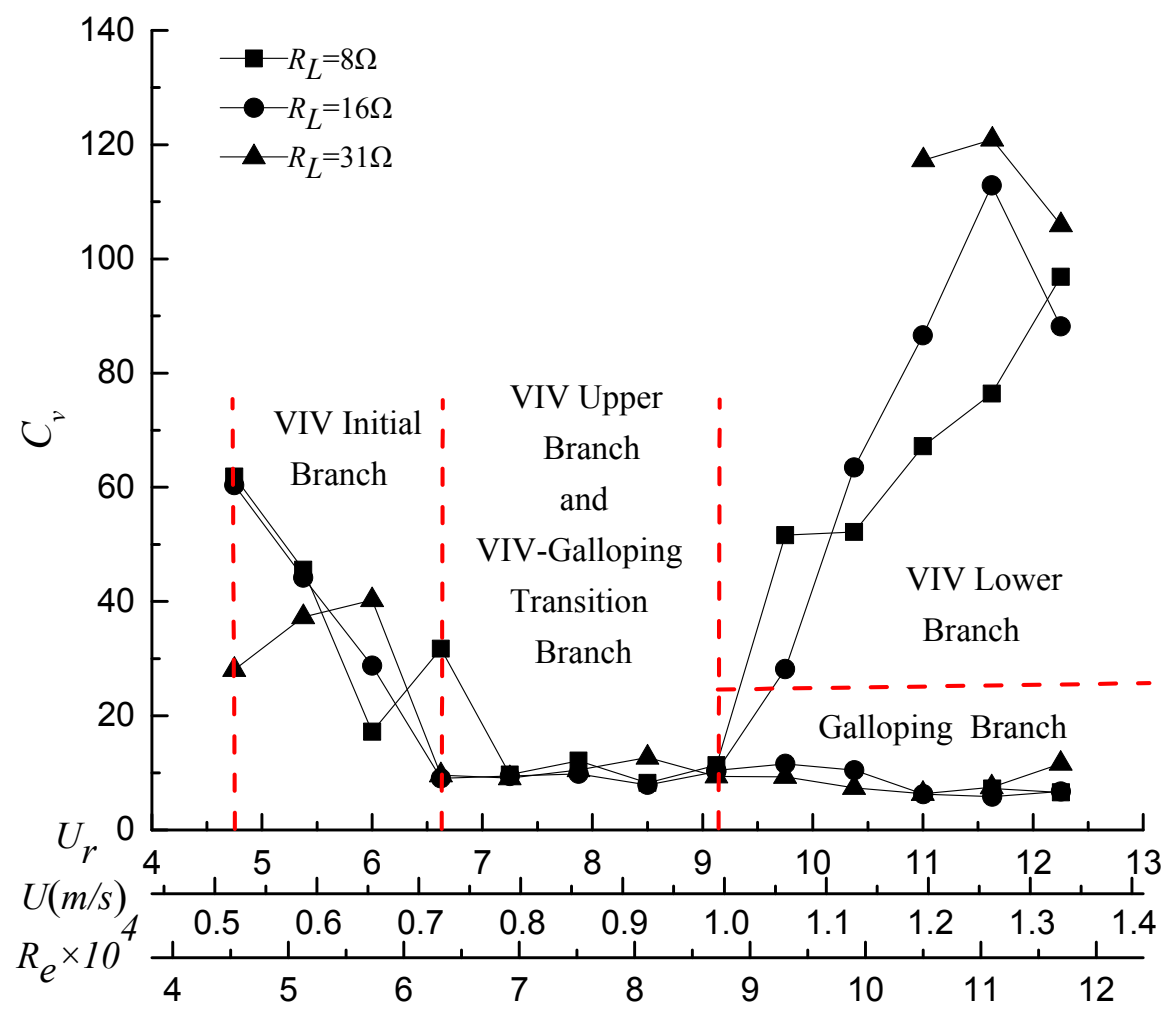

Figure 18. Power generation difference coefficient $C_{v}$ value.

For $4.75 \leq U_{r} \leq 6.625$, the value of $C_{v}$ exceeded 20 due to the unstable oscillation in the VIV initial branch, indicating that the power generation stability was poor. For $6.625 \leq U_{r} \leq 9.125$, as $U_{r}$ increased, $C_{v}$ dropped to lower than 10 and remained almost stable due to the stable oscillation of the prism. This indicated that the stability of the power output was good at the VIV upper branch. For $9.125 \leq U_{r} \leq 12.25$, two performances of $C_{v}$ could be observed. In the VIV lower branch, $C_{v}$ increased rapidly and exceeded over 100 at $U_{r}=11.625$. This indicated that the power output was more unstable than any branches, due to the poor oscillation of the prism. On the contrary, in the galloping branch, $C_{v}$ remained at approximately 10 , indicating that the power output was perfectly stable due to the perfectly stable oscillation of the prism. 
It is concluded that the galloping branch was the best of all FIM branches to obtain a stable power output. The results of the FIM power generation are summarized in Table 6.

Table 6. Power generation characteristics of different branches.

\begin{tabular}{ccccc}
\hline Branch & VIV Initial & VIV Upper & VIV Lower & Galloping \\
\hline Active Power $(\mathrm{W})$ & $0.02-1.67$ & $1.53-4.46$ & $0.12-3.92$ & $2.18-21.22$ \\
Efficiency $(\%)$ & $0.38-7.6$ & $3.45-8.9$ & $0.11-7.9$ & $4.07-20.2$ \\
Stability & bad & good & bad & best \\
\hline
\end{tabular}

\subsection{Effects of Aspect Ratios on Oscillation and Energy Conversion}

\subsubsection{Effects of Aspect Ratios on Oscillation}

Different cross-section ratios $(\alpha)$ can significantly influence the angle of attack and lift force, resulting in different performances by the FIM responses and the energy conversion of the T-section prism [43].

In this section, five tests for different $\alpha$ values were conducted. The five $\alpha$ values were $0.8,0.9,1$, 1.2 , and 1.5. In addition, the physical parameters of the system were summarized as: $m_{o s c}=29.521 \mathrm{~kg}$, $K=1200 \mathrm{~N} / \mathrm{m}$, and $R_{L}=16 \Omega$. The variation of amplitude ratio $A^{*}$ and the efficiency of $\eta_{\text {out }}$ versus the reduced velocity $U_{r}$, the incoming flow velocity $U$, and the Reynolds number $R e$ for the five $\alpha$ cases are plotted in Figures 19 and 20.

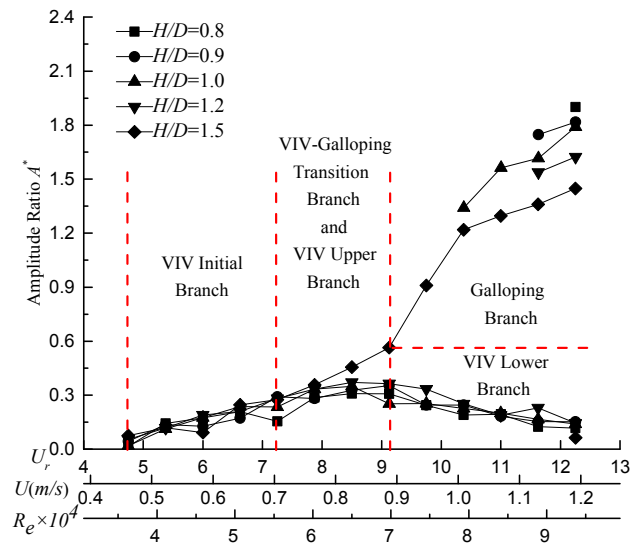

(a)

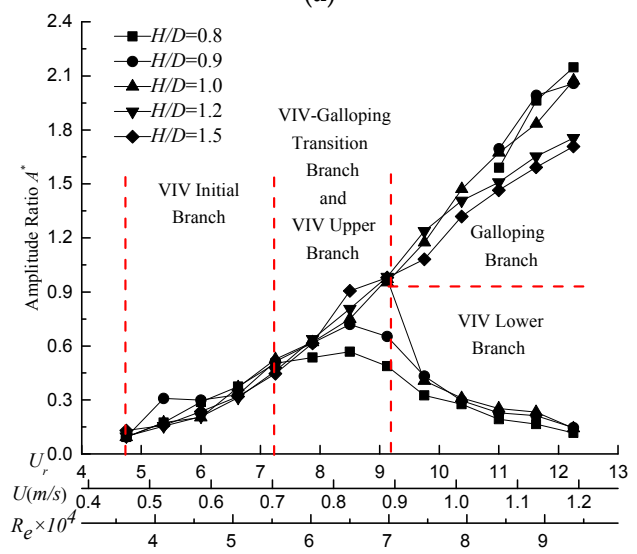

(c)

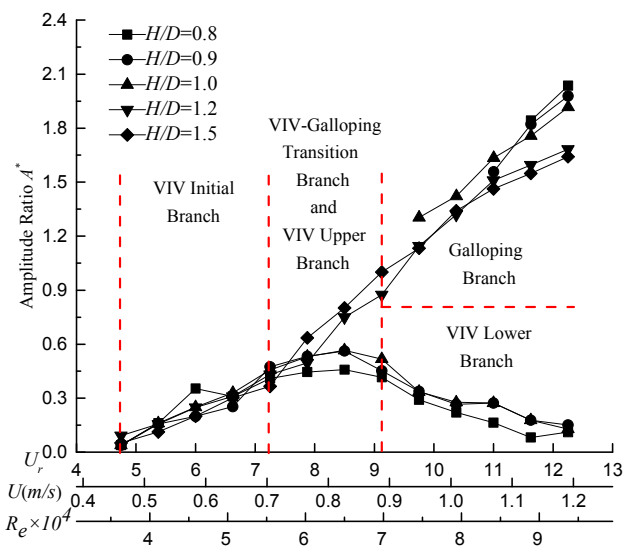

(b)

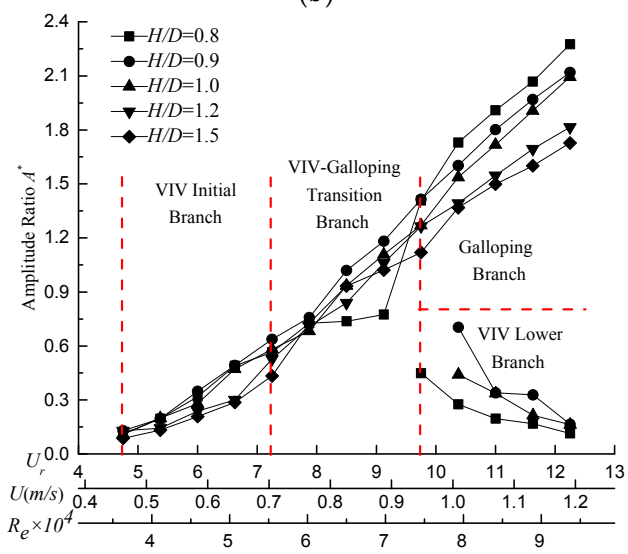

(d)

Figure 19. Oscillation characteristics with different section aspect ratios at $K=1200 \mathrm{~N} / \mathrm{m}$ : (a) amplitude ratio $(4 \Omega, \zeta=0.347)$; (b) amplitude ratio $(8 \Omega, \zeta=0.305)$; (c) amplitude ratio (11 $\Omega, \zeta=0.246$ ); (d) amplitude ratio $(16 \Omega, \zeta=0.177)$. 


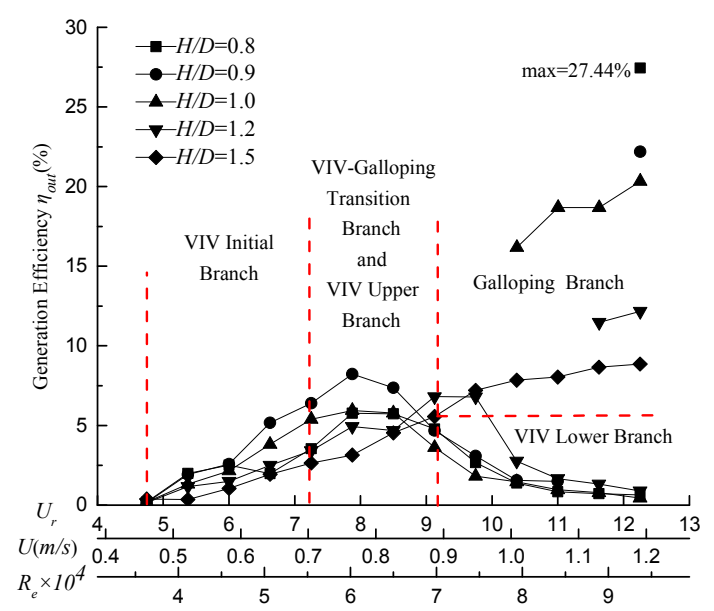

(a)

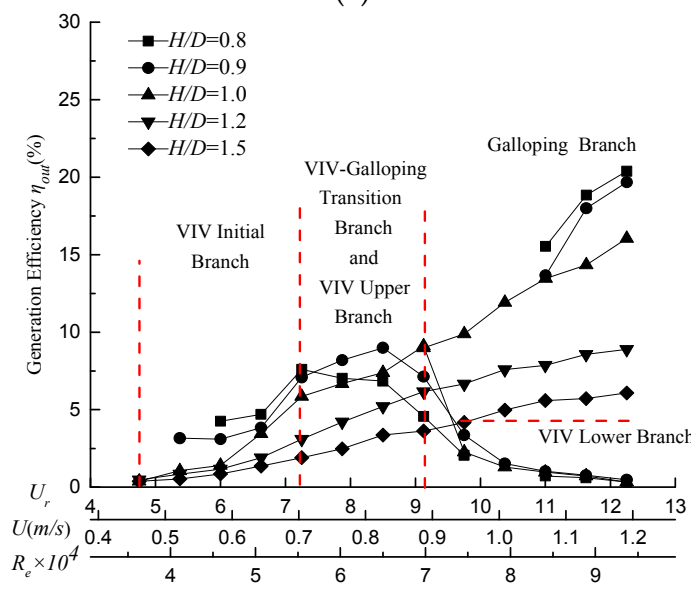

(c)

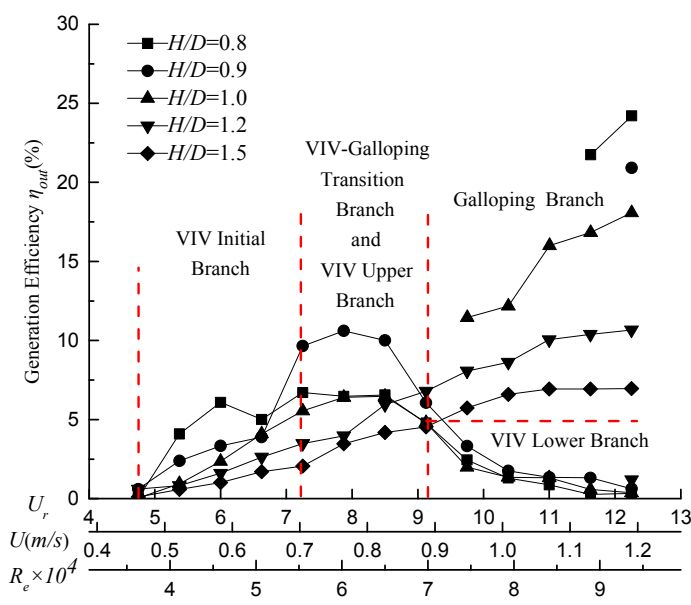

(b)

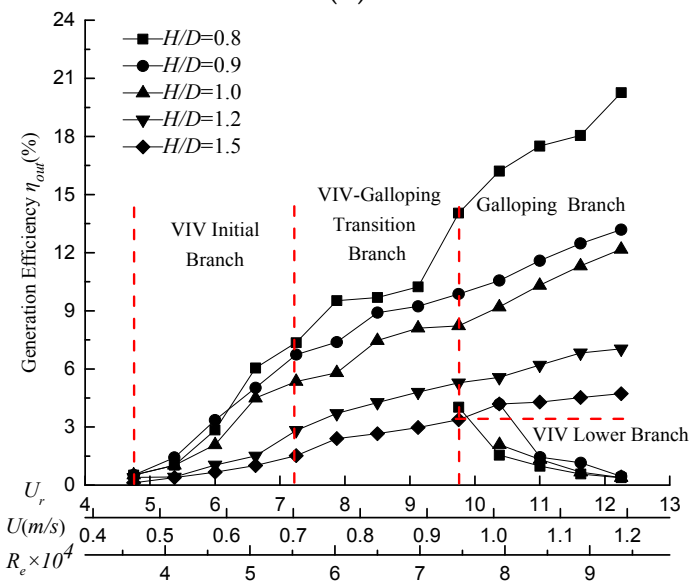

(d)

Figure 20. Power generation efficiency with different section aspect ratios at $K=1200 \mathrm{~N} / \mathrm{m}$ : (a) amplitude ratio $(4 \Omega, \zeta=0.347)$; (b) amplitude ratio $(8 \Omega, \zeta=0.305)$; (c) amplitude ratio (11 $\Omega$, $\zeta=0.246) ;(d)$ amplitude ratio $(16 \Omega, \zeta=0.177)$.

From the previous results of this paper, it was found that the T-section prism could not be self-excited from VIV to galloping at high damping conditions with $\alpha=1$. In Figure 19a, for $\zeta=0.347$, only SG occurred at $\alpha=1.5$. For the prisms of other $\alpha$ values forced by initial displacement, HG occurred at a high velocity.

In Figure 19b,c, for $\zeta=0.305$ and $\zeta=0.246$ in the prisms of $\alpha=1.5$ and $\alpha=1.2$, SG was observed, while in the prisms of other $\alpha$, HG was observed with external excitation. In Figure 19d, for $\zeta=0.177$, SG occurred under all conditions. For $1.2 \leq \alpha \leq 1.5$, the VIV lower branch could not be observed with external suppression. Based on these results, it can be concluded that a higher $\alpha$ value is more likely to cause SG under the same conditions. The rise of $\alpha$ is beneficial to the development of the oscillation mode from HG to SG. In addition, $A^{*}$ decreases with the increase of $\alpha$. Therefore, a reasonable $\alpha$ must be selected to ensure good oscillation characteristics, as well as to guarantee better electrical energy resources.

\subsubsection{Effects of Aspect Ratios on Energy Conversion}

Figure 20 presents the variation of the $\eta_{\text {out }}$ with the five $\alpha$ values, which can be summarized as follows. In the present tests, T-section prisms with a higher $\alpha$ have a good advantage at high damping conditions in terms of oscillation, but their performances in power generation efficiency are not very prominent. On the contrary, the prisms with smaller $\alpha$ show a more outstanding power generation 
advantage (shown in Figure 20). In Figure 20a, for $R_{L}=4 \Omega$, the maximum $\eta_{\text {out }}$ rises up to $27.44 \%$, which is approximately the maximum $\eta_{\text {out }}(28 \%)$ of the PTC circular cylinder. In Figure $20 \mathrm{~b}-\mathrm{d}$, it can be concluded that regardless of both high damping and low damping conditions, a smaller $\alpha$ shows a better power generation efficiency in the galloping branch. The oscillation enters the VIV lower branch, resulting in a significant decrease in $\eta_{\text {out }}$.

In summary, the optimal aspect ratio should be designed based on the oscillation branch and the flow conditions, in order to ensure reasonable energy utilization. For high stable flow velocities, the smaller the $\alpha$ value, the stronger the electric power conversion capacity. For widely variable flow velocities, a higher $\alpha$ is suitable for extracting oscillation energy from a T-section prism within a certain range of the test.

\section{Conclusions}

(1) The HG and SG of a T-section prism with external excitation are similar to those of a triangular prism. With a decrease in load resistances, the SG of the T-section prism gradually transforms to CG, and finally HG occurs. The oscillation modes of SG, HG, and CG all contain VIV branches and a galloping branch. The only difference is whether these can be self-excited from VIV to galloping. The T-section prism exhibits good stability and high intensity in the galloping branch, which is beneficial for energy extraction and utilization.

(2) The galloping branch of the T-section prism presents an efficient and stable power output in the tests. There is an optimal power generation resistance $R_{L}$ of $8 \Omega$, an optimum damping ratio $\zeta_{\text {total }}$ of 0.305 , a maximum power generation $P_{\text {harn }}$ of $21.23 \mathrm{~W}$ at $U_{r}=12.25$, and a maximum power generation efficiency $\eta_{\text {out }}$ of $20.2 \%$ at $U_{r}=11.625$. Compared with the test results of the smooth circular cylinder and the PTC circular cylinder, $\eta_{\text {out }}$ of the smooth circular cylinder is $22 \%$ [39] and the efficiency of the PTC circular cylinder is higher, at $\eta_{\text {out }}=28 \%$ and $P_{\text {harn }}=23.54 \mathrm{~W}$ [44]. In addition, the maximum $\eta_{\text {out }}$ of the T-section prism with $\alpha=0.8$ is $27.44 \%$, and it can be concluded that the T-section prism has great potential for power generation.

(3) In the present tests with aspect ratios from 0.8 to 1.5 , the smaller section aspect ratio shows a stronger ability to convert electrical energy. The aspect ratio has a great influence on the energy collection for the T-section prism. Thus, it is necessary to optimize the section aspect ratio of the prism to enhance the power generation efficiency.

Author Contributions: J.L. and X.Y. designed the experiments; X.Y., N.S., Q.R., and H.D. performed the experiments; X.Y. and N.S. analyzed the data; X.Y. built and wrote the majority of the manuscript text; all authors reviewed the manuscript; F.L. and G.X. made contributions to the structure and language of the manuscript.

Funding: This research was funded by the National Key R\&D Program of China grant number 2016 YFC0401905.

Acknowledgments: All workers from the State Key Laboratory of Hydraulic Engineering Simulation and Safety of Tianjin University are acknowledged. The authors are also grateful for the assistance of the editor and three anonymous reviewers for their professional and pertinent comments and suggestions, which were greatly helpful for further quality improvement of this manuscript.

Conflicts of Interest: The authors declare no conflict of interest.

\section{Abbreviations}

D Projection width of the prism in the direction of the incoming flow

$H \quad$ Height of the T-section prism cross-section

L Prism length

$\alpha \quad$ Section aspect ratio $H / D$

K System stiffness

$\mathrm{C}_{\text {total }} \quad$ System damping coefficient

$C_{\text {harn }} \quad$ Electromagnetic damping coefficient

$m_{\text {osc }} \quad$ Original mass, prism mass, transmission mass, and one-third of the spring mass [2]

$U \quad$ Incoming flow velocity

$U_{r} \quad$ Reduced velocity $U /\left(f_{n} \cdot D\right)$ 


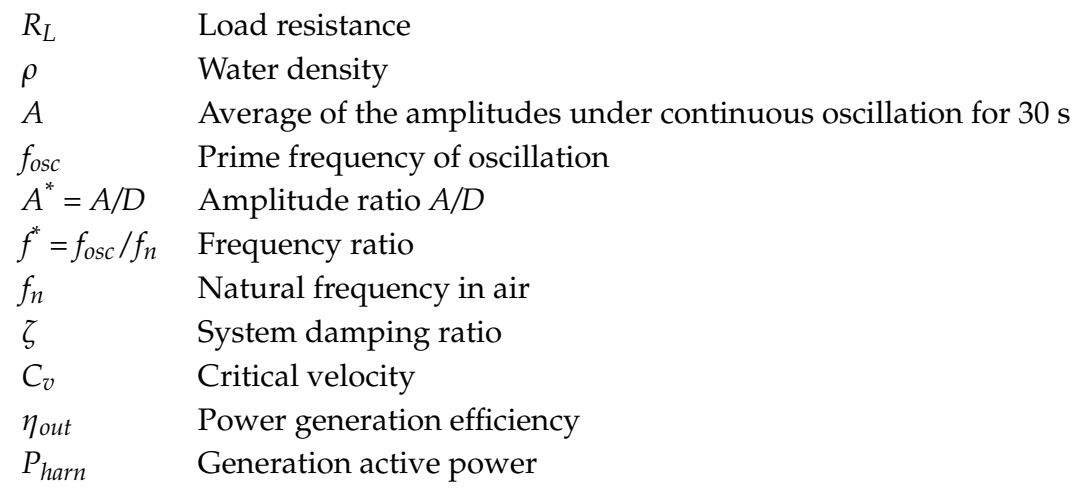

\section{References}

1. Blevins, R.D. Flow-Induced Vibration, 3rd ed.; Van Nostrand Reinhold: New York, NY, USA, 1990.

2. Barriga, J.; Ruiz-de-Gopegui, U.; Goikoetxea, J.; Coto, B.; Cachafeiro, H. Selective coatings for new concepts of parabolic trough collectors. Energy Procedia 2014, 49, 30-39. [CrossRef]

3. Cachafeiroa, H.; de Arevaloa, L.F.; Vinuesaa, R.; Lopez-Vizcaino, R.; Luna, M. Analysis of vacuum evolution inside Solar Receiver Tubes. Energy Procedia 2015, 69, 289-298. [CrossRef]

4. Cachafeiroa, H.; de Arevaloa, L.F.; Vinuesaa, R.; Goikoetxea, J.; Barriga, J. Impact of solar selective coating ageing on energy cost. Energy Procedia 2015, 69, 299-309. [CrossRef]

5. Mannini, C.; Belloli, M.; Marra, A.M.; Bayati, I.; Giappino, S.; Robustelli, F.; Bartoli, G. Aeroelastic stability of two long-span arch structures: A collaborative experience in two wind tunnel facilities. Eng. Struct. 2016, 119, 252-263. [CrossRef]

6. Seo, J.-W.; Kim, H.-K.; Park, J.; Kim, K.T.; Kim, G.N. Interference effect on vortex-induced vibration in a parallel twin cable-stayed bridge. J. Wind Eng. Ind. Aerodyn. 2013, 116, 7-20. [CrossRef]

7. Assi, G.R.; Bearman, P.W.; Tognarelli, M.A. On the stability of a free-to-rotate short-tail fairing and a splitter plate as suppressors of vortex-induced vibration. Ocean Eng. 2014, 92, 234-244. [CrossRef]

8. Dowell, E.H.; Tang, D. Nonlinear aeroelasticity and unsteady aerodynamics. AIAA J. 2002, 40, 1697-1707. [CrossRef]

9. Assi, G.R.; Bearman, P.W.; Kitney, N. Low drag solutions for suppressing vortex induced vibration of circular cylinders. J. Fluids Struct. 2009, 25, 666-675. [CrossRef]

10. Park, H.; Bernitsas, M.M.; Kumar, R.A. Selective roughness in the boundary layer to suppress flow-induced motions of circular cylinder at 30,000 $\leq \operatorname{Re} \leq 120,000$. J. Offshore Mech. Arct. Eng. 2012, 134, 041801. [CrossRef]

11. Sarpkaya, T. Fluid forces on oscillating cylinders. NASA STI/Recon Tech. Rep. A 1978, 104, $275-290$.

12. Williamson, C.H.K.; Roshko, A. Vortex formation in the wake of an oscillating cylinder. J. Fluids Struct. 1988, 2, 355-381. [CrossRef]

13. Zdravkovich, M.M. Flow around Circular Cylinders; Oxford University Press: New York, NY, USA, 1997; Volume 1.

14. De Meij, A.; Vinuesa, J.F.; Maupas, V.; Waddle, J.; Price, I.; Yaseen, B.; Ismail, A. Wind energy resource mapping of Palestine. Renew. Sustain. Energy Rev. 2016, 56, 551-562. [CrossRef]

15. Sousa, V.C.; de M Anicézio, M.; De Marqui, C., Jr.; Erturk, A. Enhanced aeroelastic energy harvesting by exploiting combined nonlinearities: Theory and Experiment. Smart Mater. Struct. 2011, 20, 094007. [CrossRef]

16. Zhang, M.; Zhao, G.; Wang, J. Study on Fluid-Induced Vibration Power Harvesting of Square Columns under Different Attack Angles. Geofluids 2017, 2017, 6439401. [CrossRef]

17. Zhou, S.; Wang, J. Dual serial vortex-induced energy harvesting system for enhanced energy harvesting. AIP Adv. 2018, 8, 075221. [CrossRef]

18. Bernitsas, M.M.; Raghavan, K.; Ben-Simon, Y.; Garcia, E.M.H. VIVACE (vortex induced vibration aquatic clean energy): A new concept in generation of clean and renewable energy from fluid flow. J. Offshore Mech. Arct. Eng. 2008, 130, 041101-041115. [CrossRef]

19. Chamorro, L.P.; Hill, C.; Neary, V.S.; Gunawan, B.; Arndt, R.E.A.; Sotiropoulos, F. Effects of energetic coherent motions on the power and wake of an axial flow turbine. Phys. Fluids 2015, 27, 055104. [CrossRef] 
20. Chamorro, L.P.; Lee, S.J.; Olsen, D.; Milliren, C.; Marr, J.; Arndt, R.E.A.; Sotiropoulos, F. Turbulence effects on a full-scale 2.5 MW horizontal-axis wind turbine under neutrally stratified conditions. Wind Energy 2015, 18, 339-349. [CrossRef]

21. Park, H.R.; Kumar, R.A.; Bernitsas, M.M. Enhancement of fluid induced vibration of rigid circular cylinder on springs by localized surface roughness at $3 \times 10^{4} \leq \operatorname{Re} \leq 1.2 \times 10^{5}$. Ocean Eng. 2013, 72, 403-415. [CrossRef]

22. Daqaq, M.F. Characterizing the response of galloping energy harvesters using actual wind statistics. J. Sound Vib. 2015, 357, 365-376. [CrossRef]

23. Hemon, P.; Amandolese, X.; Andrianne, T. Energy harvesting from galloping of prisms: A wind tunnel experiment. J. Fluids Struct. 2017, 70, 390-402. [CrossRef]

24. Alonso, G.; Meseguer, J. A parametric study of the galloping stability of two dimensional triangular cross-section bodies. J. Wind Eng. Ind. Aerodyn. 2006, 94, 241-253. [CrossRef]

25. Williamsona, C.H.K.; Govardhanb, R. A brief review of recent results in vortex-induced vibrations. J. Wind Eng. Ind. Aerodyn. 2008, 96, 713-735. [CrossRef]

26. Mannini, C.; Marra, A.M.; Massai, T.; Bartoli, G. Interference of vortex-induced vibration and transverse galloping for a rectangular cylinder. J. Fluid Struct. 2016, 66, 403-423. [CrossRef]

27. Liu, B.; Hamed, A.M.; Jin, Y.; Chamorro, L.P. Influence of vortical structure impingement on the oscillation and rotation of flat plates. J. Fluid Struct. 2017, 70, 417-427. [CrossRef]

28. Bokaian, A.R.; Geoola, F. Hydroelastic instabilities of square cylinders. J. Sound Vib. 1984, 92, $117-141$. [CrossRef]

29. Vinuesa, R.; Prus, C.; Schlatter, P.; Nagib, H.M. Convergence of numerical simulations of turbulent wall-bounded flows and mean cross-flow structure of rectangular ducts. Meccanica 2016, 51, 3025-3042. [CrossRef]

30. Lian, J.; Yan, X.; Liu, F.; Zhang, J. Analysis on Flow Induced Motion of Cylinders with Different Cross Sections and the Potential Capacity of Energy Transference from the Flow. Shock Vib. 2017, 2017, 4356367. [CrossRef]

31. Camarri, S.; Salvetti, M.V.; Buresti, G. Large-eddy simulation of the flow around a triangular prism with moderate aspect-ratio. J. Wind Eng. Ind. Aerodyn. 2006, 94, 309-322. [CrossRef]

32. Iungo, G.V.; Buresti, G. Experimental investigation on the aerodynamic loads and wake flow features of lowaspect-ratio triangular prisms at different wind directions. J. Fluid Struct. 2009, 25, 1119-1135. [CrossRef]

33. Alonso, G.; Sanz-Lobera, A.; Meseguer, J. Hysteresis phenomena in transverse galloping of triangular cross-section bodies. J. Fluid Mech. 2012, 33, 243-251. [CrossRef]

34. Zhang, J.; Liu, F.; Lian, J.; Yan, X.; Ren, Q. Flow Induced Vibration and Energy Extraction of an Equilateral Triangle Prism at Different System Damping Ratios. Energies 2016, 9, 938. [CrossRef]

35. Zhang, J.; Xu, G.; Liu, F.; Lian, J.; Yan, X. Experimental investigation on the flow induced vibration of an equilateral triangle prism in water. Appl. Ocean Res. 2016, 61, 92-100. [CrossRef]

36. Lian, J.; Yan, X.; Liu, F.; Zhang, J.; Ren, Q.; Yan, X. Experimental Investigation on Soft Galloping and Hard Galloping of Triangular Prisms. Appl. Sci. 2017, 7, 198. [CrossRef]

37. Lee, J.H.; Bernitsas, M.M. High-damping High-Reynolds VIV tests for energy harnessing using the VIVACE converter. Ocean Eng. 2011, 38, 1697-1712. [CrossRef]

38. Hai, S.; Kim, E.S.; Bernitsas, M.P.; Bernitsas, M.M. Virtual Spring-Damping System for Flow-Induced Motion Experiments. J. Offshore Mech. Arct. Eng. 2015, 137, 061801.

39. Bernitsas, M.M.; Ben-Simon, Y.; Raghavan, K.; Garcia, E.M. The VIVACE converter: Model tests at high damping and Reynolds number around $10^{5}$. J. Offshore Mech. Arct. Eng. 2009, 131, 011102. [CrossRef]

40. Chang, C.C.; Kumar, R.A.; Bernitsas, M.M. VIV and galloping of single circular cylinder with surface roughness at $3.0 \times 10^{4} \leq \operatorname{Re} \leq 1.2 \times 10^{5}$. Ocean Eng. 2011, 38, 1713-1732. [CrossRef]

41. Hai, S.; Kim, E.S.; Nowakowski, G.; Mauer, E.; Bernitsas, M.M. Effect of mass-ratio, damping, and stiffness on optimal hydrokinetic energy conversion of a single, rough cylinder in flow induced motions. Renew. Energy 2016, 99, 936-959.

42. Ma, C.; Sun, H.; Nowakowski, G.; Mauer, E.; Bernitsas, M.M. Nonlinear piecewise restoring force in hydrokinetic power conversion using flow induced motions of single cylinder. Ocean Eng. 2016, 128, 1-12. [CrossRef] 
43. Lian, J.; Ren, Q.; Liu, F.; Yan, X.; Zhang, J. Experimental Study of Flow-Induced Vibration Characteristics of T-shape Cross-Section Oscillator. J. Exp. Mech. 2017, 32, 216-222. (In Chinese)

44. Lin, D. Research on Flow Induced Motion of Multiple Circular Cylinders with Passive Turbulence Control; Chong Qing University: Chongqing, China, 2013. (In Chinese) 